\title{
Direct and indirect measurements on electrocaloric effect: Recent developments and perspectives
}

Cite as: Appl. Phys. Rev. 3, 031102 (2016); https://doi.org/10.1063/1.4958327

Submitted: 03 May 2016 . Accepted: 23 June 2016 . Published Online: 27 July 2016

Yang Liu, James F. Scott (D), and Brahim Dkhil

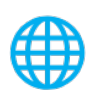

View Online

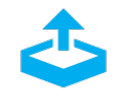

\section{ARTICLES YOU MAY BE INTERESTED IN}

Organic and inorganic relaxor ferroelectrics with giant electrocaloric effect

Applied Physics Letters 97, 162904 (2010); https://doi.org/10.1063/1.3501975

Why is the electrocaloric effect so small in ferroelectrics?

APL Materials 4, 064106 (2016); https://doi.org/10.1063/1.4950788

Comparison of directly and indirectly measured electrocaloric effect in relaxor ferroelectric polymers

Applied Physics Letters 97, 202901 (2010); https://doi.org/10.1063/1.3514255

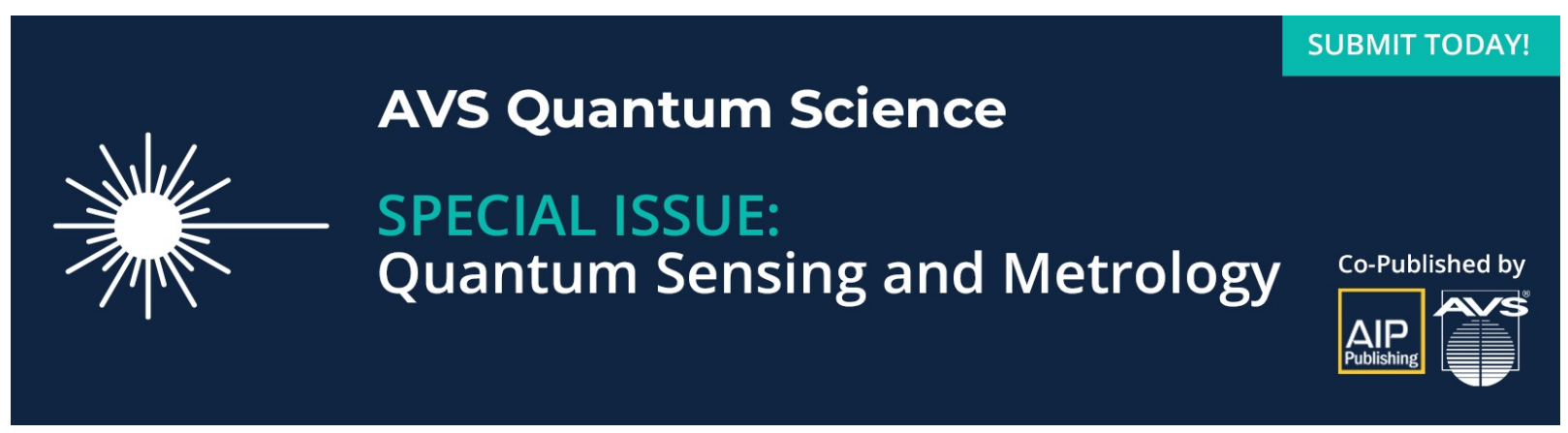




\title{
Direct and indirect measurements on electrocaloric effect: Recent developments and perspectives
}

\author{
Yang Liu, ${ }^{1, a)}$ James F. Scott, ${ }^{2,3}$ and Brahim Dkhil ${ }^{1}$ \\ ${ }^{1}$ Laboratoire Structures, Propriétés et Modélisation des Solides, CentraleSupélec, CNRS-UMR8580, \\ Université Paris-Saclay, Châtenay-Malabry Cedex 92295, France \\ ${ }^{2}$ School of Chemistry, University of St Andrews, St Andrews, Scotland, United Kingdom \\ ${ }^{3}$ School of Physics and Astronomy, University of St Andrews, St Andrews, Scotland, United Kingdom
}

(Received 3 May 2016; accepted 23 June 2016; published online 27 July 2016)

\begin{abstract}
It has been ten years since the discovery of the giant electrocaloric effect in ferroelectric materials showed that it is possible to employ this effect for substantial cooling applications. This last decade has been marked by increasing research interest, especially in characterizing and measuring the electrocaloric effect using both the so-called indirect and direct approaches. In this context, a comprehensive summary and careful reexamination of these approaches are very timely and of great importance to justify the assumptions used in different measurement techniques. This review is therefore dedicated to cover recent important and rapid advances from both the indirect and direct measurements and provides critical insights relevant for quantifying the electrocaloric effect. It involves electrocaloric materials from normal ferroelectrics, antiferroelectrics, and relaxors, and it fundamentally focuses on how the electrocaloric entropy changes in response to electric field in these typical electrocalorics. The article addresses recent developments, especially during the past three years, such as technical selection of proper polarization-electric field loops, negative electrocaloric effect in antiferroelectrics and relaxors, the controversial debate on the indirect method in relaxors, the important role of field dependence of specific heat, kinetic factors, and so on. Moreover, this review also is concerned with extracting reliable data by direct measurements. Four typical techniques and devices used recently, such as thermocouples, differential scanning calorimeters, specifically designed calorimeters, and scanning thermal microscopy, are briefly reviewed, while infrared cameras are emphasized. We hope that our review will not only provide a useful background to understand fundamentally the electrocaloric effect and what one really measures but also may act as a practical guide to exploit and develop electrocalorics towards the design of suitable devices. Published by AIP Publishing. [http://dx.doi.org/10.1063/1.4958327]
\end{abstract}

\section{TABLE OF CONTENTS}

I. INTRODUCTION $\ldots \ldots \ldots \ldots \ldots \ldots \ldots \ldots \ldots$

II. INDIRECT MEASUREMENTS. . . . . . . . .

A. Basic thermodynamic description of electrocaloric refrigeration............

B. Selection of isothermal $P-T$ curves ......

C. Negative electrocaloric effect in

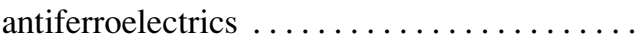

D. Electrocaloric effect in relaxors .........

E. First-order phase transition. ...........

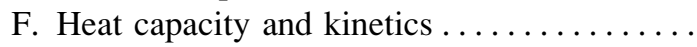

G. Depolarizing field $\ldots \ldots \ldots \ldots \ldots \ldots \ldots$

H. Clausius-Clapeyron method...........

III. DIRECT MEASUREMENTS $\ldots \ldots \ldots \ldots \ldots \ldots$

A. Thermocouple and thermometer.........

B. Differential Scanning Calorimetry (DSC) ...

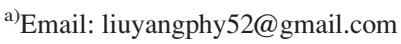

C. Specifically designed calorimeters ........ 13

D. Infra-red camera ................ 13

E. Scanning thermal microscopy .......... 15

IV. PERSPECTIVES . . . . . . . . . . . . . . . . . 15

V. CLOSING REMARKS ............... 16

2

4

5

7

8

9

10

11 
related to ferroelectric/antiferroelectric/relaxor ceramics were reported, but the best electrocaloric effect data yielded an adiabatic temperature change of less than $2.5 \mathrm{~K}$ in $\mathrm{Pb}_{0.99} \mathrm{Nb}_{0.02}\left(\mathrm{Zr}_{0.75} \mathrm{Sn}_{0.20} \mathrm{Ti}_{0.05}\right) \mathrm{O}_{3}$ ceramics in $1981 .{ }^{14} \mathrm{In}$ addition, a similar electrocaloric effect of $2.4 \mathrm{~K}$ was reported in $\mathrm{PbSc}_{0.5} \mathrm{Ta}_{0.5} \mathrm{O}_{3}$ ceramics in 2002. ${ }^{15}$ It is not until 2006 that a Science paper ${ }^{16}$ inspired both fundamental physics and exciting device applications for electrocaloric effect. Mischenko et al. at Cambridge reported a giant electrocaloric effect with an adiabatic temperature change of about $12 \mathrm{~K}$ in antiferroelectric $\mathrm{PbZr}_{0.95} \mathrm{Ti}_{0.05} \mathrm{O}_{3}$ ceramic thin films under a high electric field of $780 \mathrm{kV} / \mathrm{cm} .{ }^{16}$ Two years later, another breakthrough related to a giant electrocaloric effect in organic $\mathrm{P}(\mathrm{VDF}-\mathrm{TrFE})$ thin films was announced by the Penn State group of Zhang. ${ }^{17}$ In these two pioneering works, ${ }^{16,17}$ high electric fields could be achieved at low voltages in thin films. As a result, significantly enhanced electrocaloric response can be obtained.

Both studies ${ }^{16,17}$ employed indirect measurement to characterize the electrocaloric effects. Since then this indirect measurement, based on the Maxwell relations, has become a well-established practice in the field. ${ }^{1-12}$ This approach is useful for rapid selection of electrocaloric materials. However, uncertainties can arise when improper methods are used in such approach. In this regard, a direct measurement is quite necessary to inspire the confidence of measured electrocaloric effect for commercial device applications. ${ }^{1}$ Direct measurement means a direct determination of the electrocaloric temperature change of the sample when the external electric field is applied or removed adiabatically. Usually, the direct measurements are performed by using a thermocouple, differential scanning calorimeter (DSC), infra-red (IR) camera, scanning thermal microscopy, or specifically designed calorimeters. ${ }^{1}$ According to the literature, most of the experimental data reported are obtained by specific homemade calorimeters, and some of the data vary significantly even for the same class of materials. ${ }^{1,5}$ In this context, it is of importance to compare the results measured using different experimental techniques. For instance, commercially available techniques such as IR cameras or other calorimeters may be helpful to allow a standard practice between different groups at least at the materials level. This can avoid any overestimation or underestimation of the electrocaloric effect and would provide a reliable basis for developments of electrocaloric prototypes.

Although the goal is to arrive towards commercial applications, research in the field of electrocaloric effect is still at its early stage. Current publications mainly focus on the properties of materials, and only few works are devoted to the design of electrocaloric prototypes for applications. ${ }^{1,12}$ In contrast, significant engineering developments have been achieved in magnetocaloric refrigeration: about 60 magnetocaloric prototypes have been developed all over the world during the past two decades. ${ }^{18}$ A part of delay encountered in the field of electrocalorics is due to the way of extracting the electrocaloric data and their reliability. Therefore, it is of great importance to gain insights into the different (indirect and/or direct) approaches and address their basic characteristics. Summarizing recent developments on indirect and direct measurements on electrocaloric effect will also provide a useful background and practical guide to understand and exploit the electrocaloric effect. Therefore, this review is intending to bring an overview on the current electrocaloric measurements complementary to other excellent works, i.e., a recent book ${ }^{1}$ and other reviews, ${ }^{2-12}$ which we strongly recommend to readers. We address recent developments that were not systematically and comprehensively discussed or focused on in the previous works ${ }^{1-12}$ especially during the past three years, such as selection of polarization-electric field $P(E)$ loops, negative electrocaloric effect in antiferroelectrics, controversial debate on the indirect method in relaxors, the important role of field dependence of specific heat, kinetics factors, and so on. Our aim is to gain deeper insights into electrocaloric effect by addressing the indirect method based on Maxwell relations while emphasizing the direct measurements to set a solid foundation for developing electrocaloric prototypes.

The review is organized as follows: the first section (Section II) is devoted to indirect measurement, where basic thermodynamic descriptions of electrocalorics are presented and discussed. It includes introduction to electrocaloric refrigeration and indirect method based on the Maxwell relations, selection of the isothermal $P-T$ curves, negative electrocaloric effect in antiferroelectrics, applications of the Maxwell method to relaxors and first-order phase transitions, the role of heat capacity, kinetics, and the depolarizing field, and brief information about the Clausius-Clapeyron method. It also physically involves the typical entropy change profile with respect to electric field in the most studied electrocalorics-normal ferroelectrics, antiferroelectrics, and relaxors. The second section (Section III) concerns direct measurements. Five typical measuring techniques used recently, such as thermocouple, DSC, specifically designed calorimeters, IR camera, and scanning thermal microscopy, are briefly reviewed, while IR camera is particularly addressed. Finally, perspectives on electrocaloric studies in the future are proposed in Section IV and then the article is briefly summarized in Section V.

\section{INDIRECT MEASUREMENTS}

\section{A. Basic thermodynamic description of electrocaloric refrigeration}

There are three typical thermodynamic refrigeration cycles: Carnot, Brayton, and Ericsson cycles, which were used to present basic thermodynamic features of magnetic refrigeration. ${ }^{18}$ Here, we use electrical analog to depict refrigeration through electrocaloric Brayton-like cycles (see Figure 1), which was discussed in Scott's review ${ }^{3}$ and used in electrocaloric cooling or heat pumping devices (Refs. 19-21 and references therein). Discussions about the Ericsson-like (Ericsson cycles differ from Brayton ones mainly from the facts that external stimulus is isothermally applied or removed) ${ }^{1}$ and Carnot-like cycles (consisting of two adiabatic steps and two isothermal steps) ${ }^{22}$ can be also found elsewhere. Note that electrocaloric refrigeration based on the electrocaloric effect is reported to achieve a higher coefficient of performance (COP, defined as the ratio 


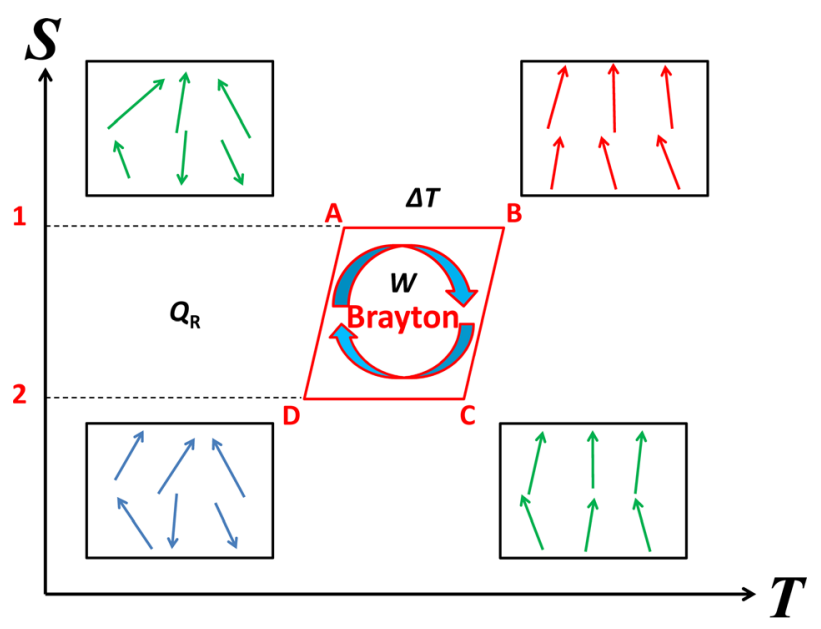

FIG. 1. Schematic of ideal electrocaloric Brayton refrigeration cycle in space of total entropy-temperature.

between the extracted heat with respect to the input work)over $50 \%$ of Carnot efficiency. ${ }^{23}$

According to Figure 1, thermodynamic Brayton cycles can be described as follows: (A-B) adiabatic application of external electric field (with no contact with any sink); (B-C) isofield cooling of the electrocaloric material (with contact with the cold sink); (C-D) adiabatic removal of the external field (with no contact with any sink); and (D-A) isofield heating of the electrocaloric material (with contact with the hot sink). The specific electrical work corresponds to the area bounded by the surface $\mathrm{A}-\mathrm{B}-\mathrm{C}-\mathrm{D}$, while the maximum extracted heat of the electrical Brayton refrigeration cycle is denoted as $Q_{\mathrm{R}}$, i.e., inside the surface 1-A-D-2. Therefore, the coefficient of performance $C O P$ can be defined as $C O P_{\mathrm{B}}=Q_{\mathrm{R}} /|W|$. Considering an ideal Carnot cycle, the
$C O P$ can be written as $C O P_{\mathrm{C}}=T_{\mathrm{R}} /\left(T_{\mathrm{H}}-T_{\mathrm{R}}\right)$, where $T_{\mathrm{R}}$ and $T_{\mathrm{H}}$ indicate the temperature of cold and hot reservoirs, respectively. The relative efficiency of a refrigerator with respect to an ideal Carnot cycle can be defined as $\eta=C O P / C O P$.

Now let us concentrate the basic thermodynamic description of the electrocaloric effect. Based on the Maxwell relationship $\left(\frac{\partial S}{\partial E}\right)_{T}=\left(\frac{\partial P}{\partial T}\right)_{E}$, the reversible electrocaloric change in temperature $\Delta T$ of an electrically responsive material due to a change in external electric field $\Delta E=E_{2}-E_{1}$ under adiabatic conditions can be determined by

$$
\Delta T=-\int_{E_{1}}^{E_{2}} \frac{T}{C(E, T) \rho}\left(\frac{\partial P}{\partial T}\right)_{E} d E,
$$

where $T$ is the temperature; $P$ is the polarization; $\rho$ is the mass density; and $C(E, T)$ is the heat capacity; all depend on both electric field and temperature. Note that another quantity called isothermal entropy change $\Delta S=\int_{E_{1}}^{E_{2}}\left(\frac{\partial P}{\partial T}\right)_{E} d E$, is equally important to describe the electrocaloric $E_{\text {effect. }}$ However, its important role is not widely recognized in the literature since $\Delta S$ is directly related to the absorbed or ejected heat in response to isothermal application/removal of electric field. ${ }^{8}$ The entropy behavior will be discussed in detail later in several typical electrocalorics.

According to Equation (1), $\Delta T$ or $\Delta S$ is usually maximized near the phase transition, where thermally driven changes in measured polarization $P$ arising from changes in the magnitude $\left(\frac{\partial P}{\partial T}\right)_{E}$ are largest. Recalling the features of phase transition, $P$ changes discontinuously at the transition temperature $T_{\mathrm{C}}$ for first-order phase transition, whereas for second order transitions it decays continuously with
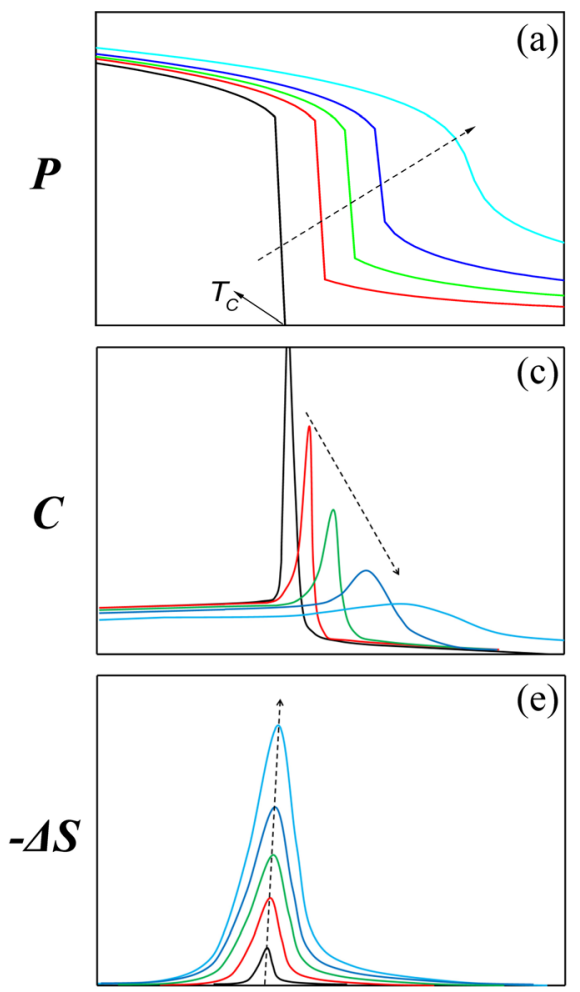

$T$

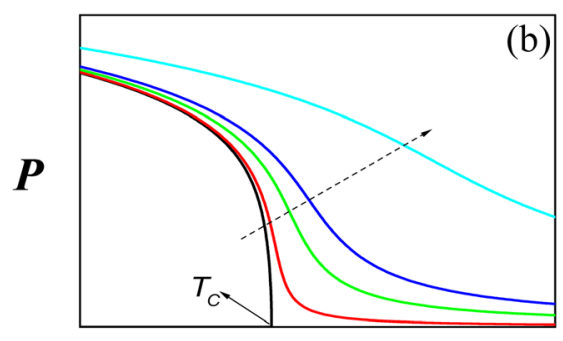

(d)

C

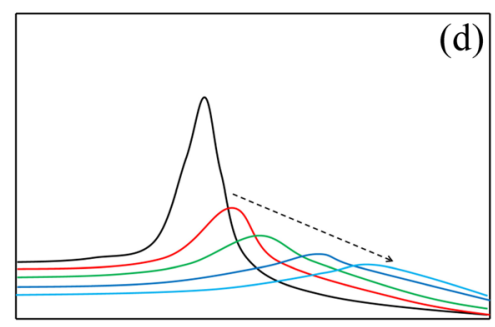

(f)

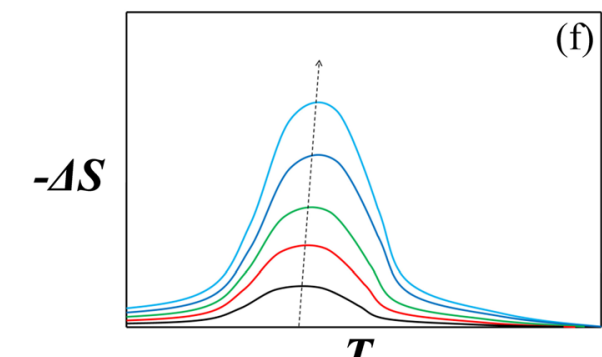

FIG. 2. Polarization versus temperature curves under various magnitudes of external electric fields: (a) firstorder phase transition and (b) secondorder phase transition. Temperature dependence of heat capacity under different electric fields for (c) first-order phase transition and (d) second-order phase transition. Isothermal entropy change as a function of temperature for (e) first-order phase transition and (e) second-order phase transition. The dashed arrows indicate the increasing trend of electric fields. 
increasing temperature until $T_{\mathrm{C}}$ (see Figures 2(a) and 2(b)). In this regard, the electrocaloric response in terms of $\Delta S$ is usually significantly stronger in materials undergoing first order phase transitions than those with second order (see Figures 2(e) and 2(f)); a tricritical point is often optimum.

As long as $P(E, T)$ is precisely known, it simply becomes a mathematical problem to calculate its derivative, its integral, and thus $\Delta T$ or $\Delta S$. This approach can naturally include the boundary conditions and/or coupling to the strains (for example, in thin films) and therefore is a standard practice in this field. ${ }^{11,24,25}$ However, in practical situations, precise determination of $P(E, T)$ and its derivative $\left(\frac{\partial P}{\partial T}\right)_{E}$ are challenging, especially for discontinuous first-order phase transitions, and cause several concerns (see Sections II B and Section IIE). Moreover, the specific heat $C(E, T)$ in Equation (1) is both temperature- and field-dependent. In particular, compared with the zero-field heat capacity $C(0)$, $C(E, T)$ usually experiences a significant change under ultrahigh electric fields, regardless of the nature of phase transition (see Figures 2(c) and 2(d)). However, $C(E, T)$ is usually taken as a constant, i.e., zero-field $C(0)$ in the literature. This unwarranted assumption may lead to serious systematic errors, which will be discussed in Section IIF. Moreover, usually application of an electric field would lead to the ordering of electrical dipoles accompanied by reduction of entropy regardless of the nature of phase transition (see Figures 2(e) and 2(f)). This thermal phenomenon corresponds to positive (conventional) electrocaloric effect. In addition, the electrocaloric response induced by a first-order phase transition is sharper than that corresponding to secondorder transition. In other words, as shown in Figures 2(e) and 2(f), the magnitude of electrocaloric effect associated with first-order phase transition can be significantly larger than that accompanied by second-order phase transition, whereas the working temperature window becomes narrower using a first-order phase transition.

\section{B. Selection of isothermal $P$ - $T$ curves}

As mentioned in Section II A, the electrocaloric effect can be derived based on the data from the temperature dependence of the polarization (isothermal $P-T$ curves) obtained either from theory or experiment. Figure 3 shows antiferroelectric $P(E)$ loops measured at room temperature. In experiments, upper branches of $P(E)$ loops $(E>0)$, in which the ferroelectric/antiferroelectric domain configurations are not significantly modified due to the large electric field, are usually selected to ensure that the indirect method based on Maxwell relations is reliable. ${ }^{16}$ In the case of lower branches, the electric field threshold is not high enough $(E>0)$ and undesired ferroelectric switching occurs. In addition, in antiferroelectrics, there may be some lowervoltage regions of the upper legs of the hysteresis cycle (which we denote as "lower" regions in Figure 3) which are also associated with polarization switching and remarkable changes in the domain configurations. The concern about using the lower hysteresis leg or this "lower" part of the upper branches to calculate $\Delta T$ is that a significantly smaller

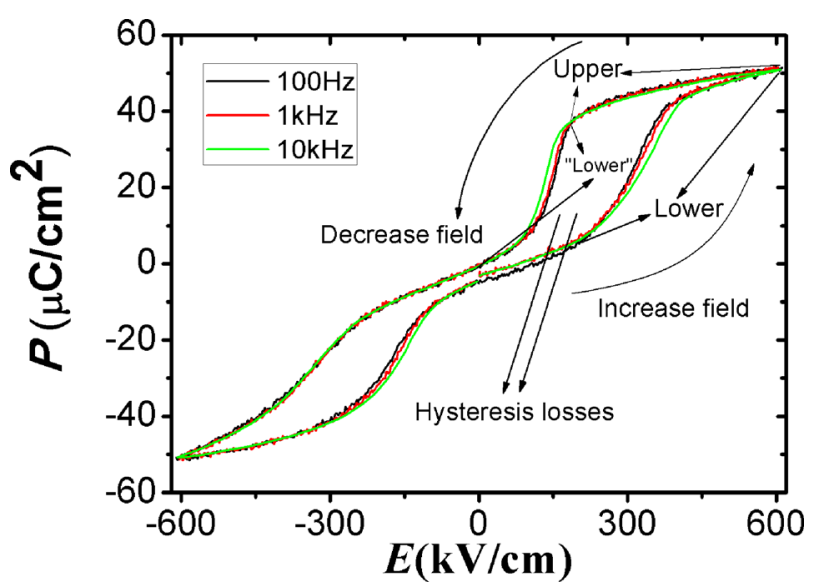

FIG. 3. $P(E)$ loops of antiferroelectric $\left(\mathrm{Pb}_{0.97} \mathrm{La}_{0.02}\right)\left(\mathrm{Zr}_{0.95} \mathrm{Ti}_{0.05}\right) \mathrm{O}_{3}$ thin films measured under different frequencies at room temperature. The single phase films were fabricated on a $\mathrm{Pt}(111) / \mathrm{Ti} / \mathrm{SiO}_{2} / \mathrm{Si}$ substrate using sol-gel spin coating and the thickness is about $650 \mathrm{~nm}$. Lower, "lower" and upper branches are specifically indicated. The arrows act as a guide to eyes. It can be seen that the frequency has a negligible influence on shape of $P(E)$ loops here.

electrocaloric effect may be predicted. For instance, experimental results in $\mathrm{BaTiO}_{3}$ single crystals showed that electrocaloric responses $(\Delta T)$ derived from the lower branches show $50 \%$ smaller values than that from the upper branches or the result from direct measurement. ${ }^{26}$

This finding was attributed to the dramatic change in ferroelectric domain configurations when a modest electric field is applied. ${ }^{26}$ However, according to the following analysis, there are at least two overlooked factors which demand further considerations: (1) The role of electric field dependence of specific heat ${ }^{24,27}$ was not taken into account. This may also lead to remarkable underestimation of electrocaloric responses when a constant heat capacity is used in Equation (1) (see Section IIF). In other words, the use of upper and lower branch $P(E)$ loops to calculate $\Delta T$ both suffer from this important factor. (2) Direct measurement results ${ }^{26,28}$ indicate that the electrical hysteresis losses may play an important role leading to an asymmetry between the electrocaloric heating (lower branches with electrical field increasing) and the cooling responses (upper branches corresponding to decreasing electric field). To be more specific, if the upper branches (excluding the "lower" regions in Figure 3 ) correspond to the intrinsic electrocaloric cooling (precluding the contributions from ferroelectric switching and hysteresis losses) when the field is removed, the lower branches in turn would reflect the extrinsic contributions from the hysteresis losses to the electrocaloric heating; the change in domain configuration (growth or switching) is responsible for the $P(E)$ hysteresis loops. As a result, the electrocaloric heating temperature change (lower branches) may move through the cooling peak temperature (upper branches). This was observed in recent direct measurements on organic thick films ${ }^{28}$ and also $\mathrm{BaTiO}_{3}$ single crystals. ${ }^{26}$ Therefore, we argue that the concern about $\Delta T$ by using lower branches could arise as a result of hysteresis losses. As hysteresis losses strongly depend on the magnitude of external fields, and their contributions to extract $\Delta T$ become less dominant with decreasing field. This evidence implies that remarkable 
discrepancies between upper and lower branches can only be observed under high electric fields. As a result, it implies that reasonable estimations can be obtained even though a significant domain configuration change exists, i.e., near the antiferroelectric-to-ferroelectric switching regions. ${ }^{29}$ In addition, Joule heating under high fields can also result in a similar asymmetry. ${ }^{30}$ As addressed in the review, ${ }^{3}$ the materials will get hotter and hotter if the Joule heating is dominant, and no cooling effect will be obtained asymptotically.

We also note that even the ferroelectric switching in ferroelectrics or antiferroelectrics is a complex phenomenon, and the electrocaloric effect during ferroelectric switching is poorly understood since it is related to kinetic factors. Indeed, the ferroelectric switching mechanism is usually described by nucleation of new domains, forward growth followed by their sideways growth. Yet in many materials, nucleation is the rate-limiting step. It therefore still requires more efforts to provide solid experimental evidence or theoretical insights. In order to avoid ferroelectric switching, Crossley suggested an alternative approach using proper $P(E)$ loops. ${ }^{31}$ However, factors such as applied frequency are not taken into account and analyzed. Indeed, the shape of $P(E)$ loops also depends on the measured frequency (see Figure 3), which is often ignored in the literature and will be discussed in Section II F.

$P(E)$ loops are measured at a constant temperature and for different temperatures when the sample is cooled or heated. In standard practice, fourth (or sixth)-order polynomial $^{16}$ or Gaussian fittings ${ }^{17}$ to $P-T$ curves are carried out to infer $\partial P / \partial T$ in Equation (1). It is usually required that the temperature interval should be small enough to ensure that isothermal $P-T$ curves are as smooth as possible. Otherwise, very strange electrocaloric responses might be obtained, such as remarkable shifts of electrocaloric peak positions, significant variation in the values of predicted $\Delta T$ introduced by different smoothing approaches, and erroneous prediction of electrocaloric peaks even without any phase transitions. ${ }^{32}$

Special concern for antiferroelectrics when selecting $P-T$ curves is to exclude the contributions from isentropiclike polarization rotations. ${ }^{29}$ To be more specific, in the antiferroelectric region $\left(E_{1}=0 \mathrm{kV} / \mathrm{cm}\right)$, Equation (1) actually not only takes into account the contribution arising from the change in the magnitude of the staggered polarization $P_{\mathrm{A}}=P_{\mathrm{a}}-P_{\mathrm{b}}{ }^{33}$ (where $P_{\mathrm{a}}$ and $P_{\mathrm{b}}$ are the polarizations of two sublattices) but also that resulting from the polarization rotations driven by changes in the directions of the dipoles being not collinear with the electric field. The former part is the truly physical contribution to the entropy change, while the later plays a negligible role even though it may be accompanied by a modest polarization rise during the initial application of a low electric field (well below the antiferroelectric-ferroelectric transition field $\left.E_{\mathrm{AFE}-\mathrm{FE}}\right)$. Therefore, Equation (1) may overestimate the electrocaloric effect $\left(E_{1}=0 \mathrm{kV} / \mathrm{cm}\right)$ in antiferroelectrics, since it artificially includes the contribution from isentropic-like polarization rotation during the initial poling process $\left(E_{1} \ll E_{\mathrm{AFE}-\mathrm{FE}}\right)$, which should be subtracted. ${ }^{29}$ One common treatment using Equation (1) is to deliberately apply a high electric field $E_{1}$ (much larger than antiferroelectric-ferroelectric transition field $\left.E_{\mathrm{AFE}-\mathrm{FE}}\right)$ to stabilize the ferroelectric regime. ${ }^{16}$ After that, a larger electric field $E_{2}>E_{1}$ is applied to induce the positive (conventional) electrocaloric response. In this case, using the upper branches subjected to high electric fields might avoid the controversy using of lower branches proposed in Ref. 26. However, the phase transition from antiferroelectric phase to ferroelectric phase is not employed to optimize the electrocaloric response in antiferroelectrics simply because it is energetically too expensive and inefficient.

\section{Negative electrocaloric effect in antiferroelectrics}

Recent publications in antiferroelectric materials either simply followed the foregoing strategy $E_{1}>E_{\mathrm{AFE}-\mathrm{FE}}$ (Refs. 16,34 , and 35 and references therein) or studied the electrocaloric response for the case of $E_{1}=0$ (Refs. 29, 32, and 36-42 and references therein) in order to use the electricinduced antiferroelectric-to-ferroelectric phase transition. However, recent findings $\left(E_{1}=0\right)$ in the literature appear controversial with respect to positive or negative electrocaloric effect. A negative electrocaloric effect means materials will cool rather than warm in the case of positive electrocaloric effect when an electric field is applied adiabatically, whereas they warm when the field is withdrawn adiabatically. Specifically, a giant positive electrocaloric effect of $45.3 \mathrm{~K}$ at $17^{\circ} \mathrm{C}$ (it is always positive within the temperature of interest) was predicted in antiferroelectric $\mathrm{Pb}_{0.8} \mathrm{Ba}_{0.2} \mathrm{ZrO}_{3}$ thin films using indirect measurements, ${ }^{37}$ while a moderate negative electrocaloric effect (about $-0.5 \mathrm{~K}$ to $-1.5 \mathrm{~K}$ depending on the field) was observed by direct measurements in prototypical $\mathrm{PbZrO}_{3}{ }^{41}$ and $\mathrm{Pb}_{0.94}$ $\mathrm{Ba}_{0.06} \mathrm{ZrO}_{3}{ }^{42}$ ceramics near room temperature. A negative electrocaloric effect was predicted in $(\mathrm{Na}, \mathrm{Bi}) \mathrm{TiO}_{3}$-based ceramics by indirect measurements, ${ }^{36,40}$ while recent direct measurements on similar ceramic compositions near the morphotropic phase boundary showed significant discrepancies between the direct and indirect electrocaloric measurements. ${ }^{43}$ Moreover, a giant negative electrocaloric effect (about $-5 \mathrm{~K}$ ) was demonstrated in La-doped $\mathrm{Pb}\left(\mathrm{Zr}_{0.95} \mathrm{Ti}_{0.05}\right) \mathrm{O}_{3}$ thin films $(0.65 \mu \mathrm{m})$ near room temperature using indirect method, ${ }^{29}$ while only positive electrocaloric effect was reported in La-doped $\mathrm{Pb}\left(\mathrm{Zr}_{0.85} \mathrm{Ti}_{0.15}\right) \mathrm{O}_{3}$ thin films $(1 \mu \mathrm{m})$ within the whole temperature of interest including both room temperature and higher temperatures. ${ }^{39}$ These inconsistencies in the literature may be probably attributed to (1) the unjustified nature of antiferroelectricity [some $P(E)$ loops even show evidence of ferroelectricity or slim relaxor type!] without further evidence of microscopic structure, and (2) inaccurate fits of $\left(\frac{\partial P}{\partial T}\right)_{E}$ without considering the field- and temperature dependent specific heat and measurement frequency (see Section IIF). The first concern may be a key reason why only positive electrocaloric effects were observed in antiferroelectric compounds using indirect measurements. The second concern may likely account for the quantitative discrepancies between direct and indirect measurements. 
As mentioned in Section II B, it may be risky to use Equation (1) in antiferroelectrics where the transition is between antiferroelectric and ferroelectric phases. Moreover, simply choosing the measured (average) polarization as the primary ferroic order parameter may be questionable since the relevant order parameter in the antiferroelectric state is the staggered polarization rather than the average polarization. ${ }^{33}$ While very similar concerns about the magnetic order parameters in antiferromagnetics were raised by Gschneidner et al., ${ }^{44}$ treating the measured magnetization as the primary order parameter is frequently used in the magnetocaloric community. ${ }^{45-52}$ It seems that the indirect method might still yield reasonable estimations about direct measurement results. For instance, it was reported that the direct measurement results in FeRh compounds can be well reproduced by the indirect method based on the magnetization measurements. ${ }^{52}$

The entropy change $\Delta S(E)$ under an applied field in an antiferroelectric is not always negative, as shown in Figures 2(e) and 2(f) in normal ferroelectric ( $E$ does not increase the existing antiferroelectric order). This may lead to a negative electrocaloric effect, changing our general understanding of the electrocaloric effect. ${ }^{29,32,41,42}$ One can already realize that, for the case of normal ferroelectrics (Figures 2(e) and 2(f)), applying a (conjugate) field $E$ always reduces entropy by further aligning dipoles, therefore leading to a conventional (positive) electrocaloric effect, according to Equation (1) and recalling that $\left(\frac{\partial S}{\partial E}\right)_{T}=\left(\frac{\partial P}{\partial T}\right)_{E}$. Therefore, this process should always be accompanied by a decrease in entropy, as shown in Figures 2(e) and 2(f). A negative electrocaloric effect, however, indicates that electrocaloric entropy increases when an external electric field is applied isothermally and becomes smaller when the field is withdrawn.

Indeed, being able to observe a negative electrocaloric effect in antiferroelectric under a moderate electric field is reasonable and understandable if one recalls the magnetic analog (called the negative magnetocaloric effect) which was found in antiferromagnetic FeRh alloys 26 years ago. ${ }^{45}$ In order to understand physically this electrically induced thermal effect in antiferroelectrics, using the Kittel model, Pirc et al. predicted a crossover temperature above which the low-temperature negative electrocaloric effect transforms into high-temperature positive type. ${ }^{41}$ This was qualitatively confirmed by recent experimental works in antiferroelectric thin films ${ }^{29}$ together with Young's pioneering work. $^{32}$ Moreover, a possible mechanism was proposed to explain the origin of the negative electrocaloric effect in antiferroelectrics when subjected to a modest electric field under isothermal conditions. ${ }^{29}$ To be more specific, the non-collinear responses of dipoles in antiferroelectrics to an applied electric field lead to the increase of the entropy components perpendicular to the direction of the electric field with the entropy component along the direction of electric field nearly unchanged (see Figure 4). As a result, the application of a modest electric field in antiferroelectrics will generally increase the entropy by misaligning (canting) the dipoles, therefore generating the negative electrocaloric effect. ${ }^{29} \mathrm{We}$ also note that this collective non-collinear response of (a)

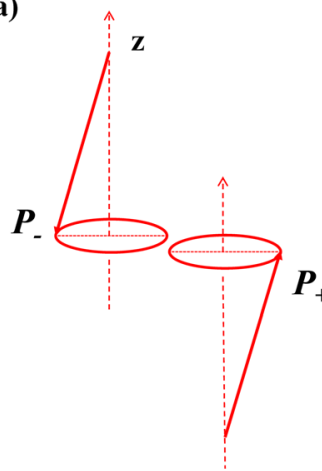

(b)

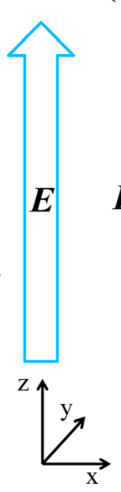

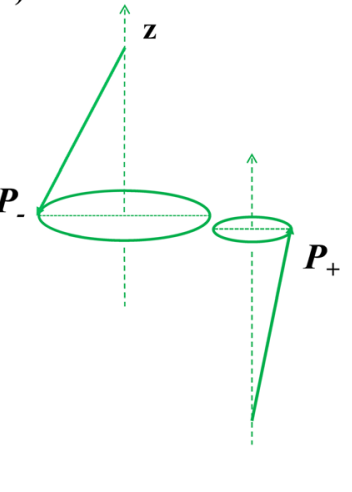

FIG. 4. Schematic of a possible mechanism of negative electrocaloric effect in antiferroelectric: (a) without any electric field and (b) under a modest electric field. Due to temperature fluctuations, local polarization $P$ locally moves around, roughly in cones. The dipoles are collinear only on average and the cones have the same radii without any field.

antiferroelectric dipoles is different from the initial random arrangement of dipoles as aforementioned.

Furthermore, since both antiferroelectric state and ferroelectric state are ordered with nominally different order parameters, the existence of negative electrocaloric effect in antiferroelectrics seems to be reasonable. Entropy clearly plays the key role in electrocaloric properties. Let us qualitatively analyze the electric-field-induced entropy change in antiferroelectrics as depicted in Figure 5 as follows: It is known that a ferroelectric order can be always stabilized from an antiferroelectric state and more easily at relatively higher temperatures and higher electric fields. ${ }^{29,33}$ Without any external fields, the antiferroelectric state is stabilized with a nominal entropy $S_{\mathrm{AFE}}(E=0)$ (which is deliberately set to be a constant $S_{0}$ in our following analysis). When the electric field is applied isothermally $\left(E_{1}=0\right)$, local antiferroelectrically arranged dipoles will rotate and align along the direction of electric field until local phase switches from antiferroelectric domain configuration to ferroelectric-like type in the antiferroelectric matrix, assuming a first-order phase transition. In this case, the whole system becomes "disordered" or less ordered with an intermediate entropy $S_{\mathrm{AFE}-\mathrm{FE}}$, which is larger than $S_{0}$. This provides a simple and

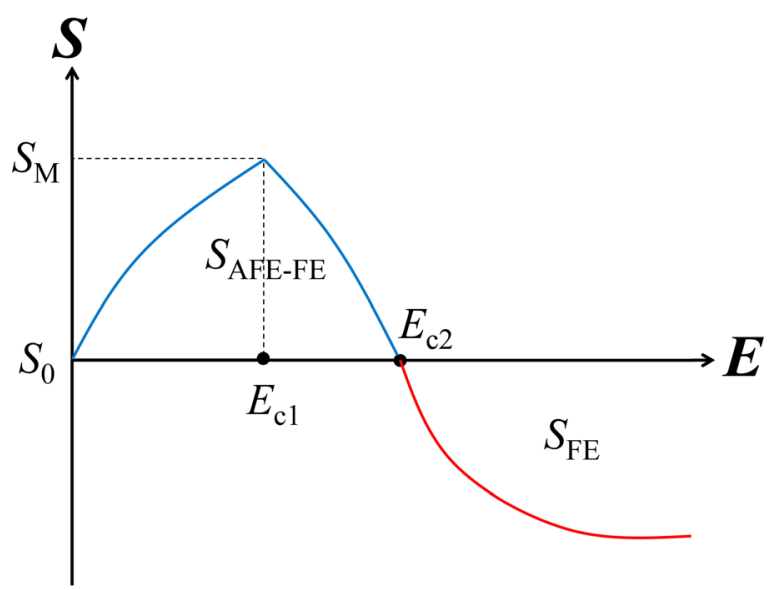

FIG. 5. Schematic of the principle of the electric-field-induced entropy change in antiferroelectrics. 


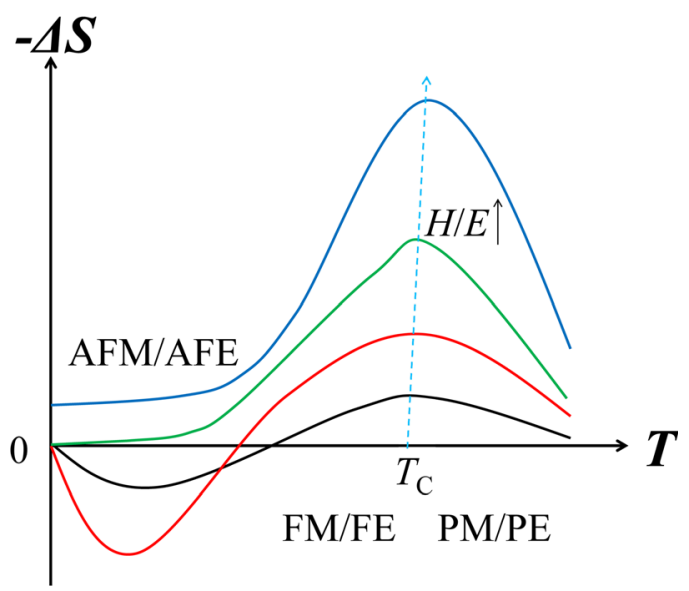

FIG. 6. Schematic of the principle about the temperature dependence of magnetocaloric/electrocaloric entropy change under various magnetic/electric fields. AFM/AFE indicates antiferromagnetic/antiferroelectric, FM/FE refers to ferromagnetic/ferroelectric, and $\mathrm{PM} / \mathrm{PE}$ indicates paramagnetic/ paraelectric, respectively. $T_{\mathrm{C}}$ is the Curie temperature corresponds to the high-temperature transition from $\mathrm{PM} / \mathrm{PE}$ phase to FM/FE phase. At the low temperature, AFM/AFE is stabilized without any fields.

common physical image for the origin of negative electrocaloric effect in antiferroelectrics under a modest electric field. The upper bound magnitude of negative electrocaloric response $S_{\mathrm{M}}$ is limited by the competition between antiferroelectric and ferroelectric orderings. With the electric field up to a critical electric field $E_{\mathrm{c} 1}\left(\approx E_{\mathrm{AFE}-\mathrm{FE}}\right)$, the entropy contribution from ferroelectric order becomes comparable with its antiferroelectric counterpart. The electric field decreases the antipolar order while increasing the polar one. This means that antipolar entropy contribution increases, whereas the polar one decreases. In this regard, the whole system becomes ordered again, accompanied by a decrease in the entropy $S_{\mathrm{AFE}-\mathrm{FE}}$. The entropy behavior ends within a ferroelectric regime $S_{\mathrm{FE}}\left(E>E_{\mathrm{c} 2}\right)$ where the negative electrocaloric effect is completely compensated by the positive electrocaloric one and the sign of the electrocaloric effect changes to positive.

The physical description proposed above is not only restricted in understanding the thermal behavior in antiferroelectrics but can be also useful to explain the non-monotonic magnetocaloric behavior in antiferromagnetic materials when subjected to field-induced phase transition from an antiferromagnetic phase to a ferromagnetic phase. ${ }^{48-51}$ To simplify, in Figure 6, we do not consider any specific information such as the material and the direction of fields but focus on the general physical behavior of an antiferroic system. The magnetocaloric or electrocaloric entropy change $\Delta S$ with a positive sign at lower temperatures $\left(\ll T_{\mathrm{C}}\right)(1)$ first experiences an increase in its magnitude (becoming more positive) with magnetic or electric field increasing; (2) $|\Delta S|$ then decreases when a critical magnetic or electric field is reached; and (3) $\Delta S$ changes its sign from positive to negative, which indicates that the nature of magnetocaloric or electrocaloric effect changes from negative type to positive type (also see positive electrocaloric entropy change in Figures 2(e) and 2(f)). In the higher temperatures near the Curie temperature, entropy change is always negative indicating positive caloric response due to the field induced transition from a paraelectric or paramagnetic phase to a ferroelectric or ferromagnetic phase.

Regarding antiferroelectrics, the analysis above implies a subtle coupling between antiferroelectric and ferroelectric polarizations, and their structural instabilities might be at the heart of this complex mechanism. ${ }^{53}$ Indeed, recent firstprinciples calculations predicted the existence of a so-called scaling law of $\Delta T$ for the fields below $E_{\mathrm{AFE}-\mathrm{FE}}$ in $\mathrm{PbZrO}_{3} .{ }^{54}$ However, the computational results failed to reproduce several typical experimental behaviors: ${ }^{29,32}$ the remarkable shift of polarization peak with temperature under various electric fields, the shift of negative $\Delta T$ peak with temperature under various electric fields, and the sign reversal under ultrahigh electric fields. In addition, relaxor ferroelectics were also found to display a modest negative electrocaloric effect in a limited temperature range, which will be discussed in Section IID. The potential interest for negative electrocaloric or magnetocaloric effect is not only to develop a new class of caloric materials in solid-state caloric family but also to enhance the cooling efficiency if both negative and positive caloric effects can be combined properly.

\section{Electrocaloric effect in relaxors}

Relaxors are characterized by strong dielectric constant anomalies in which temperature of the susceptibility maximum depends on the measurement frequency. It is believed that polar nanoregions cause such anomalies and that a classical long-range ferroelectric state is avoided because of the presence of random electric fields. There is still debate on the validity of electrocaloric data from the indirect approach based on the Maxwell relations especially for these relaxor ferroelectric materials, because they are not in thermal or mechanical equilibrium. For instance, it was reported that the indirect method using Equation (1) may not necessarily yield a reliable basis for electrocaloric response in the relaxors. ${ }^{43,55-58}$ Note that the relaxors are known to be not mechanical, polar, or thermal equilibrium and therefore it is technically unjustified to use the Maxwell relations in relaxors whose $S(E)$ is generally unknown. The interest of relaxors for electrocaloric studies originated from the following considerations: The magnitude of $\Delta T$ in relaxors is highly competitive with that in normal ferroelectrics; ${ }^{1,5}$ the operational temperature window can be quite wide; ${ }^{17,55-58}$ a dual broad peak was observed $;^{59-63}$ there are small electrical and thermal losses; ${ }^{17}$ and the non-equilibrium state of polar phase and the coexistence of numerous polar phases may provide multiple sources of entropy change especially near the critical composition, ${ }^{64,65}$ which is also applicable in normal ferroelectric solid solutions with critical point. ${ }^{66-68}$

Recently, a theoretical model without the use of Maxwell relations was proposed to describe the electrocaloric effect in relaxor ferroelectrics. ${ }^{69}$ The total entropy of a polar material was divided into two parts: one is dipolar degrees of freedom or polar nanoregions, which corresponds to polar contributions and thus is electric-field-dependent. The other component results from the lattice, which is a field-independent contribution (phonons, electrons, etc). Another assumption is that the dipolar free energy can be 
written in the standard Landau form. Therefore, following the thermodynamic relations as described in Ref. 69, the electrocaloric effect $\Delta T=T_{2}-T_{1}$ in response to an applied field $E$ can be implicitly derived from: ${ }^{69}$

$$
T_{2}=T_{1} \exp \left\{\frac{1}{2 C_{\text {latt }}}\left[a_{1}\left(T_{2}\right) P^{2}\left(E, T_{2}\right)-a_{1}\left(T_{1}\right) P^{2}\left(0, T_{1}\right)\right]\right\},
$$

where $C_{\text {latt }}$ is the lattice heat capacity per unit volume and assumed to be nonsingular and dependent weakly on the temperature. $a_{1}=d a / d T$, where $a$ is a temperature dependent Landau free energy expansion coefficient. As a result, this model can explain the maximum electrocaloric strength observed near the critical point where the first-order phase transition transforms into second-order type (tricritical point) as the field increases ${ }^{27,58,69,70}$ and is also applicable to normal ferroelectrics. ${ }^{70}$ In addition, based on this framework, theoretical descriptions of the elastocaloric ${ }^{71}$ and barocaloric effects $^{72}$ associated with uniaxial stress and hydrostatic pressure were reported recently. The interest in these predictions is to demonstrate the promise of ferroelectrics in mechanocaloric cooling applications together with recent and rapid developments in this field (Refs. 73-82 and references therein). Indeed, this model (see Equation (2)) can be also used to justify the negative electrocaloric effect found in relaxors as long as the parameters such as polarization and heat capacity are precisely known. Efforts in relaxors require further investigations. For instance, from the theoretical point of view, first-principles calculations combined with non-equilibrium molecular dynamics can be used to determine the electrocaloric effect without the use of Maxwell relations. $^{83}$

It was reported that single crystals of $\mathrm{PbMg}_{1 / 3} \mathrm{Nb}_{2 / 3} \mathrm{O}_{3^{-}}$ $\mathrm{PbTiO}_{3}$ (PMN-PT) were also found to display a negative electrocaloric effect. ${ }^{62,84}$ For instance, a negative electrocaloric effect with a quite small magnitude of about $0.025 \mathrm{~K}$ was first evidenced around $60^{\circ} \mathrm{C}$ in $\langle 011\rangle$-oriented PMN28PT single crystals under an electric field of $9 \mathrm{kV} / \mathrm{cm}^{62}$ Later on, $\langle 001\rangle$-oriented PMN-30PT single crystals were studied in detail by both indirect measurement and direct measurement based on DSC. ${ }^{84}$ The findings confirmed the existence of negative electrocaloric effect near a relatively narrow region $\left[75-95^{\circ} \mathrm{C}\right]$. Moreover, both the indirect and direct measurement results agree with each other within the experimental errors, even for relaxors. ${ }^{84}$ The origin of this effect in relaxors was theoretically studied by using a one dimensional statistical mechanical lattice model, and the change in electrocaloric sign was attributed to the variation in free energies driven by electric field. ${ }^{85}$ Nevertheless, in contrast to these results, no negative electrocaloric response was found in ceramics made of the same material PMN30PT. ${ }^{58}$

According to recent theoretical developments in relaxors [for instance, $\mathrm{Ba}\left(\mathrm{Zr}_{\mathrm{x}} \mathrm{Ti}_{1-\mathrm{x}}\right) \mathrm{O}_{3}$ relaxors], there is a dielectric peak around a specific temperature, $T_{\mathrm{m}}$, associated with the application of weak dc electric fields. ${ }^{86}$ No macroscopic paraelectric-to-ferroelectric transition occurs in this material down to the lowest possible temperatures. Such behavior therefore implies that the (induced) polarization decreases as the temperature decreases below $T_{\mathrm{m}}$ when applying these weak fields, which thus should result in the negative electrocaloric effect according to Equation (1). Finally, theoretical insights using $a b$ initio calculations are highly desired to understand particularly the origin of the negative electrocaloric effect in antiferroelectrics and relaxors.

The microscopic model we described in Section II C may be applied to relaxors. Indeed, relaxors can be seen as a similar system where the polar state should fight against the random fields (rather than antiferroic interactions).

\section{E. First-order phase transition}

Strictly speaking, Equation (1) is not valid in the case of first-order phase transitions (these are not thermodynamically reversible ${ }^{6}$ ). Recalling the phase diagram in the space of $(P, T)$, the polarization exhibits a discontinuous change at a critical temperature $T_{C}$ corresponding to the first-order phase transition (Figure 2(a)). Both $\left(\frac{\partial P}{\partial T}\right)_{E}$ and heat capacity (Figure 2(c)) are rather challenging to define. In experiments, the phase transition usually occurs in a specific temperature range with phase coexistence. Latent heat referring to the heat energy rejected or absorbed within the coexistence range of two phases is an import factor describing the firstorder phase transition, whose contribution to electrocaloric response fails to be taken into account in Equation (1). ${ }^{87}$ Typically, a noticeable thermal hysteresis is often observed when the first-order phase transition is driven by cooling and heating processes. ${ }^{24}$ However, few experimental studies were conducted to consider this aspect of the electrocaloric response; ${ }^{88}$ most of the results were obtained through a single thermal path by either cooling or heating the samples. In the ideally isothermal condition, the electrical hysteretic losses should also be considered when the field is applied and removed. In this case, the relaxor has a positive characteristic due to its $\operatorname{sim} P(E)$ loops, which are not as sensitive to temperature compared with the normal ferroelectrics. ${ }^{1}$ On the contrary, the hysteresis loss in antiferroelectrics could be problematic in addition to its great volume change near the antiferroelectric-to-ferroelectric phase transition. ${ }^{29,33}$

Both the thermal and electrical losses contribute to the final electrocaloric response. Moreover, if these two factors are dominant, the electrocaloric effect may be irreversible and it would exist for only a few cycles. This degradation ("fatigue") is surely detrimental to any cooling applications. Similar issues have attracted much more attention for magnetocaloric refrigeration, ${ }^{12,89-94}$ while investigation on electrocaloric counterpart is in its infancy stage. ${ }^{1-12}$ Quantitative analysis of contributions of thermal and electrical losses to the electrocaloric effect is of great importance especially for the design of electrocaloric prototype devices.

According to the analysis above, great caution should be made to include every detailed information regarding the discontinuity in $P(E, T)$ associated with a first-order phase transition when one may try to fit $P(E, T)$ with some polynomials. ${ }^{11,32}$ This is challenging. Taking into account the discontinuous change of the polarization at the phase 
transition, electrocaloric entropy change $\Delta S$ was suggested to be modified as follows: ${ }^{1,6}$

$$
\Delta S=\int_{0}^{E}\left(\frac{\partial D}{\partial T}\right)_{E} d E-\Delta D\left(\frac{\partial E}{\partial T}\right) .
$$

From Equation (3), the Maxwell relation can be expressed in two forms: one in which $d S$ is proportional to $d E$, and the other form in which $d S$ is proportional to the change of the displacement $d D$ having a proportional factor of $\left(\frac{\partial E}{\partial T}\right)$. Note that Equation (3) is also an approximate expression and it seems technically complex; it has not been employed in experimental work yet.

First-principles-based simulations were carried out to test the applicability of the Maxwell relations. Ponomareva and Lisenkov demonstrated that the indirect approach based on the Maxwell relations provides an accurate and reliable basis for electrocaloric effect as long as Equation (1) is accurately integrated. ${ }^{95}$ To the contrary, according to Rose and Cohen's computations, Equation (1) is an exact expression and both indirect and direct approaches must agree within the error of experiments or simulations but not necessarily depend on detailed numerical integration techniques. ${ }^{96}$ Note that both simulations ${ }^{95,96}$ started from the first-order phase transition; however, neither of them took into account the field- and temperature-dependent heat capacity nor reported any dissipative non-equilibrium effects resulting from latent heat associated with the first-order phase transitions. These important factors were included in a more recent computational framework $^{87}$ that demonstrated that the indirect approach can only be valid as long as the first order transition is not crossed. They also found that systematic errors by using indirect approach may arise due to inaccurate fits of $\left(\frac{\partial P}{\partial T}\right)_{E}$ (as we discussed above) and the use of a constant specific heat which we will address in Section II F. The theoretical approach ${ }^{87}$ by Marathe et al. represents an important advance to quantify the electrocaloric effect by using ab initio calculations. One practical route to further theoretical explorations is suggested to carry out theoretical simulations which can be directly compared with the experimentally available data.

\section{F. Heat capacity and kinetics}

Assuming a constant value of heat capacity $C(E, T)$ in Equation (1) despite the phase transition is often used in the literature since this treatment may still yield a reasonable estimate of the electrocaloric effect. ${ }^{8}$ Indeed, the heat capacity measured at zero electric field can be significantly different from those at modest and strong fields. This fieldand temperature-dependence of heat capacity may raise concern about the simple treatment widely used in the literature. Previous thermodynamic calculations ${ }^{24}$ showed that heat capacity $C(E, T)$ peak (or divergence) near the phase transition in normal ferroelectric (like $\mathrm{BaTiO}_{3}$ ) thin films will be smeared at high electric fields with a shift in its peak position towards higher temperatures (see Figure 2(c)). This behavior in normal ferroelectric $\mathrm{BaTiO}_{3}$ is confirmed by the first principles-based calculations very recently. ${ }^{87}$ In addition, the field-dependence of heat capacity in $\mathrm{Ba}_{\mathrm{x}} \mathrm{Sr}_{1-\mathrm{x}} \mathrm{TiO}_{3}$ (x from 0 to 1) at various temperatures was also calculated using Green's functions and the results imply that the value of heat capacity decreases remarkably with increasing field. ${ }^{97}$

Moreover, it is known that latent heat associated with the first-order phase transition will be fully released as long as the field is high enough. This indicates that a smearing of phase transition from first-order to second-order shape should be observed by experiments. As a result, the heat capacity peak should decrease significantly since the latent heat is determined by the area of this peak, which was experimentally observed in $\mathrm{KDP}^{98}$ and theoretically predicted in $\mathrm{BaTiO}_{3}{ }^{24,87}$ This behavior is also confirmed in $\mathrm{BaTiO}_{3}{ }^{27,70}$ and PMN-PT ${ }^{99,100}$ single crystals by using a high-resolution calorimeter with ac and relaxation modes. According to these recent calorimetric measurements, together with foregoing theoretical findings, it is reasonable to conclude here that the heat capacity is not constant but strongly dependent on the electric field and also changes with temperature.

Let us now reexamine the assumption that heat capacity is electric-field independent. According to the analysis above (see Figures 2(c) and 2(d)), the relation $C(E, T)<C(0, T)$ is usually satisfied, and any discrepancies become more significant in the case of ultrahigh electric field which drives the giant electrocaloric effect near the phase transition. The real physical contribution of heat capacity to the integral in Equation (1) is overestimated. Therefore, $\Delta T$ estimated using $C(0, T)$ or $C(0)$ should be smaller than the true electrocaloric response. This simple treatment can lead to underestimations of the real $\Delta T$, which was ignored by most of the studies in the literature. ${ }^{1}$ Indeed, even for quasi-direct measurement using DSC, numerous concerns exist when heat data are used to calculate $\Delta T$ according to the simple relation $T \Delta S=C(0) \Delta T$, which will be discussed in detail in Section III B. Given that field-dependent heat capacity can be also a source of underestimations of $\Delta T$, further works are highly desired to determine the role of ferroelectric switching, fielddependent heat capacity, and hysteresis loss.

Regarding the lower branches of $P(E)$ loops mentioned above, it is related to a very complex dynamic process associated with domain switching and growth mechanisms. ${ }^{101}$ Indeed, the Maxwell relations approach gives trends in the caloric effects since it assumes thermal equilibrium, but most of the experiments are time-dependent. The kinetics is rarely mentioned in the literature. Indeed, the use of the Maxwell relations may cause an overestimation on average of approximately $40 \%$ (see Ref. 102). The first recognition of the time and frequency dependence of the electrocaloric effect was independently made in Ref. 102 and Chapter 7 of a recent book. ${ }^{1}$ The important point is that the polarization $P(t, f)$ is relaxational with time $t$. And the hysteresis loops are usually recorded within a millisecond or less (the frequency is typically $1 \mathrm{kHz}$ or even larger), probably corresponding to the condition between isothermal and adiabatic regime which is neither isothermal nor adiabatic. This likely leads to systematic errors of $\Delta T$, which was justified and reexamined in detail by Young. ${ }^{32}$ Moreover, the instrument temperature response also has an integration time and normally these two times are not the same. In such a case, the 
Maxwell relations must be explicitly time dependent. Another concern about Equation (1) is the different relaxation times for heat capacity and polarization; these often differ and both are different from the rise time of the applied field in the experiments. Hence, the electrocaloric response depends upon three characteristic times. Future device optimization must frequency-match the detector response time and the polarization decay time.

It is known that shape of $P(E)$ loops depends on measurement frequency ${ }^{101}$ and the frequency has an important effect on $P_{\max }$ in a specific electric field and thus the calculated $\Delta T$. This complicates the calculations of $\Delta T$ or $\Delta S$ based on Equation (1) compared with the magnetocaloric analog, whereas the dc magnetic field does not have a frequency. For instance, as shown in Figure 3, the measurement frequency has a negligible influence on $P(E)$ loops and therefore results in a trivial effect on the predicted $\Delta T$ in antiferroelectric PLZT $\left[\left(\mathrm{Pb}_{0.97} \mathrm{La}_{0.02}\right)\left(\mathrm{Zr}_{0.95} \mathrm{Ti}_{0.05}\right) \mathrm{O}_{3}\right]$ thin films. ${ }^{29}$ On the contrary, $P(E)$ loops of $\mathrm{BaTiO}_{3}$ single crystals show a remarkable dependence on measured frequency at $100^{\circ} \mathrm{C}$ (near the Curie temperature of about $130^{\circ} \mathrm{C}$ ). ${ }^{103}$ As a result, the predicted $\Delta T$ based on Equation (1) depends on the frequency, and the nominal $\Delta T$ is larger at higher frequencies. Recalling the foregoing concern about $P(E)$ loops, it is therefore risky to reach the conclusion that electrocaloric effect in $\mathrm{BaTiO}_{3}$ single crystals is strongly dependent on electric field frequency. Moreover, it was recently suggested by Crossley et al. that sufficiently low frequency measurement might be useful to achieve near isothermal conditions and was reported to support the validity of the indirect method even in relaxors. ${ }^{104}$ However, $P(E)$ loops at other frequencies were not reported and similar comparison of loops (see Figure 3) between different frequencies is lacking. In this context, careful reexamination of the experimental data is strongly recommended in the future studies.

In addition, deeper insights into the contributions of ferroelectric switching, ${ }^{105,106}$ domain growth, ${ }^{107,108}$ and domain wall $^{109-112}$ to the electrocaloric effect are highly desired in order to provide basic understandings. For instance, a recent study using non-adiabatic direct measurement on PZT-5 ceramics showed that $\Delta T$ displays a hysteresis behavior with $E$ and the sign of electrocaloric effect reverses at the coercive field where polarization switching occurs. ${ }^{105}$ Using a phase field method, similar $\Delta T-E$ curves were predicted in $\mathrm{PbTiO}_{3}$ nanoparticle in which vortex domain switching was considered. ${ }^{106}$ Moreover, phase field theory predicted a sign reversal of electrocaloric response at the domain walls $\left(180^{\circ}\right.$ in Ref. 107 and $90^{\circ}$ in Ref. 108). However, this does not necessarily indicate that the coexistence of both negative and positive electrocaloric effects can really exist. The existence of remarkable $\Delta T$ gradient across the domain walls will immediately lead to fast heat exchange between different domains, and therefore only a purely positive or negative electrocaloric effect should be observed with a significantly reduced $\Delta T$.

\section{G. Depolarizing field}

It is known that in magnetocaloric refrigeration, the magnetocaloric response usually needs to be corrected by taking

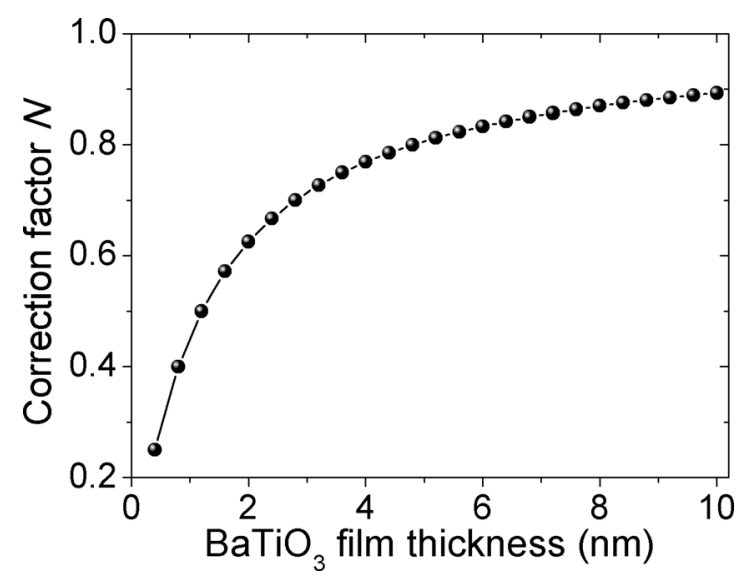

FIG. 7. The theoretical results on the thickness dependence of the correction factor $N$ originated from the depolarizing field in epitaxial (001)-oriented $\mathrm{BaTiO}_{3}$ thin films.

into account the demagnetizing field. ${ }^{113-117}$ Considering the case of the depolarizing field in ferroelectrics, its influence on electrocaloric properties is ignored in most studies. This is because for strongly polar materials, such as ferroelectrics, $D \sim P$, one can have $\left(\frac{\partial D}{\partial T}\right)_{E} \approx\left(\frac{\partial P}{\partial T}\right)_{E}$ and therefore may validate the use of Equation (1). However, this is not the case for ultrathin films in which the depolarizing field due to incomplete screening from the electrode has to be taken into account. ${ }^{118}$ The electrocaloric effect in ultrathin ferroelectric films was systematically studied by taking into account the film thickness, electrode, mechanical stress, and depolarizing field. ${ }^{77,119-121}$ For instance, Liu et al ${ }^{77}$ found that such approximation is only valid for a film thickness $h$ being relatively big $(h>4.8 \mathrm{~nm})$. The correction factor $N$ (see Figure 7) describing the deviation between the two derivatives such that $\left(\frac{\partial D}{\partial T}\right)_{E}=N\left(\frac{\partial P}{\partial T}\right)_{E}$ is introduced. ${ }^{77} N$ can be written as $N=1-\frac{2 \varepsilon_{1} \lambda}{h \varepsilon_{0}+2 \lambda \varepsilon_{\mathrm{b}}}$ where the second term arises exactly from the contribution of depolarizing field ${ }^{77,119-121}\left(\varepsilon_{\mathrm{b}}\right.$ is the background dielectric constant, and $\varepsilon_{0}$ is the permittivity of free space. $\lambda$ is the effective screening length of ferroelectric/electrode interface). When $h$ decreases down to the critical thickness $h_{\mathrm{c}}, N$ experiences a sharp drop, which clearly indicates that the effect of the depolarizing field becomes dominant.

Tuning the electrocaloric effect by adjusting the magnitude of the residual depolarizing field has been reported recently in $\mathrm{Pb}\left(\mathrm{Zr}_{0.4} \mathrm{Ti}_{0.6}\right) \mathrm{O}_{3}$ nanodots by Prosandeev et al. ${ }^{83}$ Similarly, the depolarizing field is found to play a major role in determining the electrocaloric properties and is especially detrimental for the thinner films since it cancels out a large part of the external electric field. ${ }^{77}$ This conclusion is reached by considering only the single domain configuration. Moreover, Glazkova et al. revealed a positive effect of residual depolarizing field on the electrocaloric effect, which is attributed to formation of nanodomain in ultrathin ferroelectric thin films. ${ }^{122}$ Also note that the strict Maxwell relation $\left(\frac{\partial S}{\partial E}\right)_{T}=\left(\frac{\partial D}{\partial T}\right)_{E}$ was also frequently employed to derive $\Delta T$
in organic materials (Refs. 17 and 55 and references therein], while there are also some studies using the relation $\left(\frac{\partial D}{\partial T}\right)_{E}$ $\approx\left(\frac{\partial P}{\partial T}\right)_{E}$ even in nanoscale organic films. ${ }^{123}$ The difference 


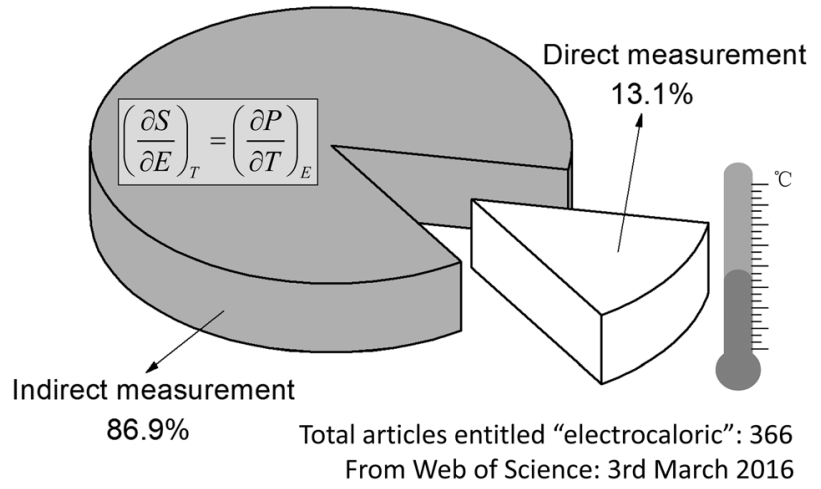

FIG. 8. Statistic result on the electrocaloric publications including direct and indirect measurements according to the Web of Science.

in calculating $\Delta T$ originating from these two different relations was unknown since it was not explicitly discussed in these works (Refs. 17, 55, and 123 and references therein].

\section{H. Clausius-Clapeyron method}

In addition to indirect method based on the Maxwell relationships, the Clausius-Clapeyron method can be also used for first-order phase transitions. ${ }^{8}$ This approach represents a nominally equivalent indirect method as that based on the Maxwell relationships, and it remains rarely employed to extract the electrocaloric effect in the literature. Moreover, the Clausius-Clapeyron equation can be used to evaluate the contributions from the latent heat of the first order phase transition which is not included in Equation (1). ${ }^{87}$ In this approach, the temperature dependence of equilibrium transition temperature $T_{0}$ and then values of entropy changes for the fully driven transition can be obtained using the Clausius-Clapeyron equation below (Equation (4)), in which order parameter changes across first-order phase transitions are denoted by the subscript " 0 " 8

$$
\Delta S=\Delta D_{0}\left(d T_{0} / d E\right)^{-1}
$$

\section{DIRECT MEASUREMENTS}

At first glance, the direct approach measurement can inspire more confidence for the design of electrocaloric devices. However, in practical cases, there are several factors making it difficult to achieve perfect adiabatic conditions. This difficulty in using the direct method can be readily seen according to the statistic result obtained from Web of Science as summarized in Figure 8. Specifically, experimental studies using direct measurements only comprise less than $15 \%$ (about 50 articles) of the total articles entitled "electrocaloric," while most works (about 85\%) were conducted by using indirect measurements. Most works are carried out to search for new materials and improve their properties, whereas only a few concepts for electrocaloric refrigeration and heat pump have been proposed (see the review in Ref. 12 or Chapter 10 in Ref. 18). These data not only imply that direct measurement is challenging but also clearly reflect the current research stage of the electrocaloric effect. This is also one reason why indirect measurements

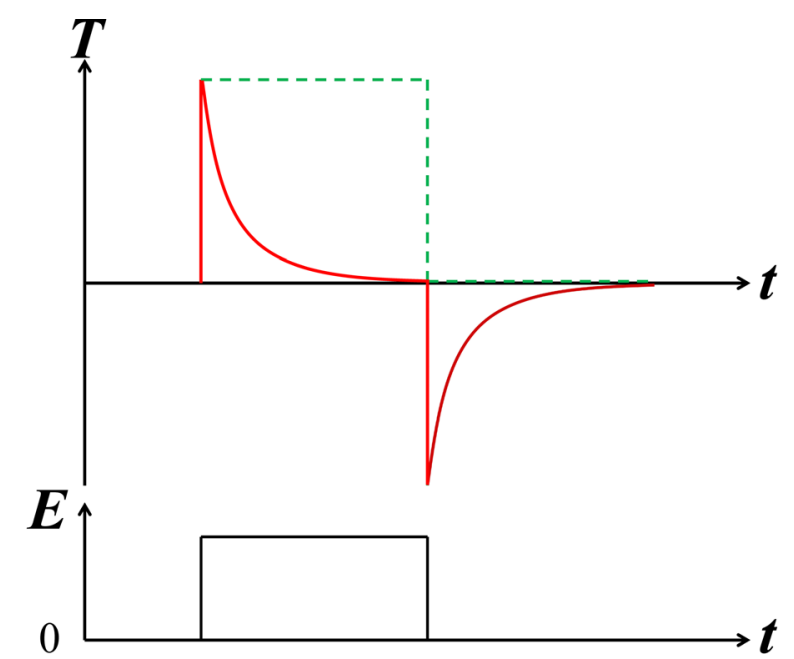

FIG. 9. Typical schematic of electrocaloric response (red line) as a function of time measured directly under near adiabatic conditions; the dashed green line indicates the ideal electrocaloric response under perfectly adiabatic conditions.

dominate this review. Because the topic of electrocaloric effect is increasing interest since 2006 (see Figure 1 in Ref. 8 ), Figure 8 also underlines the need to pursue more efforts into direct measurements and design of electrocaloric cooling devices. Sections II A-IIH have provided critical insights into the indirect measurements, which should be useful to understand and quantify the electrocaloric effect. Regarding the direct measurements which have been reviewed in detail in the previous publications, ${ }^{1,5,11}$ here we only give a brief overview of several direct techniques in order to avoid unnecessary repetition. Specifically, we will mainly focus on IR camera.

Typical result by direct measurement (such as thermocouple or thermometer, specifically designed calorimeter, IR camera, scanning thermal microscopy, and so on) is schematically shown in Figure 9. The thermal isolation of the sample is the key factor in the direct measurements. For instance, in perfectly adiabatic conditions, the sample temperature should remain constant after the electrocaloric effect is activated (dashed line in Figure 9), and the electric field is applied or removed instantaneously in contrast to the isothermal measurement carried out under nearly equilibrium conditions. However, in practical situations, the thermal exchange between the electrocaloric materials and the surroundings always exists, which leads to exponential decay of the electrocaloric peak with measurement time (see solid line in Figure 9). In this case, it is usually required that the characteristic time constant for the application (or removal) of the field should be significantly smaller than the time constant for heat exchange between sample and ambient environment in order to achieve near adiabatic conditions. This requirement can be met in bulk in most cases but fails in thin films with substrates, which makes direct determination of electrocaloric temperature change extremely challenging in thin films. As a result, it can be seen in Table I that only two experimental results were reported on nanoscale thin films ${ }^{124,125}$ during the past ten years, while most other works carried out direct measurement on thick films with a 
TABLE I. Comparison of typical results by direct measurement on electrocaloric effect in thin and thick films.

\begin{tabular}{|c|c|c|c|c|c|c|c|}
\hline Material & $h(\mu \mathrm{m})$ & $T\left({ }^{\circ} \mathrm{C}\right)$ & $\Delta E(\mathrm{kV} / \mathrm{cm})$ & $\Delta T(\mathrm{~K})$ & $|\Delta T / \Delta E|(\mathrm{K} \mathrm{cm} / \mathrm{kV})$ & Method & Reference \\
\hline$\left(\mathrm{Pb}_{0.86} \mathrm{La}_{0.08}\right)\left(\mathrm{Zr}_{0.65} \mathrm{Ti}_{0.35}\right) \mathrm{O}_{3}$ & 0.45 & 45 & 1200 & 40 & 0.033 & $\mathrm{SC}$ & 124 \\
\hline $\mathrm{PbZr}_{0.8} \mathrm{Ti}_{0.2} \mathrm{O}_{3}$ & 0.15 & 25 & 67 & 0.1 & 0.0015 & $\mathrm{SC}$ & 125 \\
\hline $\mathrm{BaZr}_{0.2} \mathrm{Ti}_{0.8} \mathrm{O}_{3}$ & 12 & 40 & 97 & 4.9 & 0.051 & $\mathrm{SC}$ & 126 \\
\hline $0.9 \mathrm{PMN}-0.1 \mathrm{PT}$ & 13 & 25 & 105 & 0.23 & 0.0022 & SThM & 104 \\
\hline$\left(\mathrm{Cd}_{0.83} \mathrm{~Pb}_{0.17}\right)_{2} \mathrm{Nb}_{2} \mathrm{O}_{7}$ MLCs & 45 & -179 & 100 & 0.8 & 0.008 & $\mathrm{SC}$ & 127 \\
\hline $\mathrm{Pb}\left(\mathrm{Sc}_{0.5} \mathrm{Ta}_{0.5}\right) \mathrm{O}_{3} \mathrm{MLCs}^{\mathrm{a}}$ & $64-72$ & 18 & 125 & 3.5 & 0.028 & $\mathrm{SC}$ & 15 \\
\hline Doped $\mathrm{BaTiO}_{3}$ MLCs & 6.5 & 47 & 300 & 0.5 & 0.0017 & TC & 128 \\
\hline $\mathrm{BaTiO}_{3}$ MLCs & 1.4 & 80 & 176 & 1.8 & 0.010 & DSC & 129 \\
\hline $\mathrm{BaTiO}_{3}$ MLCs & 1.4 & 80 & 800 & 7.1 & 0.0089 & DSC & 130 \\
\hline $\mathrm{P}(\mathrm{VDF}-\mathrm{TrFE}) 68 / 32 \mathrm{~mol} . \%$ & $10-15$ & 33 & 1600 & 20 & 0.0125 & SC & 124 \\
\hline $\mathrm{P}(\mathrm{VDF}-\mathrm{TrFE}-\mathrm{CFE})^{\mathrm{b}}$ & $4-6$ & 30 & 1500 & 16 & 0.011 & SC & 131 \\
\hline $\mathrm{P}(\mathrm{VDF}-\mathrm{TrFE}-\mathrm{CFE})^{\mathrm{c}}$ & 50 & 27 & 1000 & 4 & 0.004 & DSC/IR & 132 \\
\hline$P(V D F-T r F E-C F E)^{d}$ & $11-12$ & 25 & 900 & 5.2 & 0.0058 & IR & 133 \\
\hline Terpolymer/BNNSs/BST67 & 6 & 30 & 2500 & 50.5 & 0.0202 & SC & 134 \\
\hline $\mathrm{P}(\mathrm{VDF}-\mathrm{TrFE}) / \mathrm{BST}^{\mathrm{f}} 5^{\mathrm{f}}$ & 80 & 79 & 600 & 2.5 & 0.0042 & DSC & 135 \\
\hline Terpolymer ${ }^{\mathrm{g}} / \mathrm{PMN}-\mathrm{PT}$ & $7-15$ & 30 & 1800 & 31 & 0.017 & $\mathrm{SC}$ & 136 \\
\hline Polymer ${ }^{\mathrm{h}} /$ graphene & 11 & 25 & 400 & 5.2 & 0.013 & SC & 137 \\
\hline
\end{tabular}

${ }^{\mathrm{a}} \mathrm{Pb}\left(\mathrm{Sc}_{0.5} \mathrm{Ta}_{0.5}\right) \mathrm{O}_{3}$ was modified by doping $\mathrm{Co}$ and $\mathrm{Sb}$.

${ }^{\mathrm{b}}$ The terpolymer P(VDF-TrFE-CFE) content is 59.2/33.6/7.2 mol. \%.

${ }^{\mathrm{c}}$ The terpolymer P(VDF-TrFE-CFE) was purchased from Piezotech SA with the composition not reported.

${ }^{\mathrm{d}}$ The terpolymer P(VDF-TrFE-CFE) content is 62.6/29.4/8 mol. \%.

${ }^{\mathrm{e}}$ Terpolymer refers to the relaxor ferroelectric $\mathrm{P}(\mathrm{VDF}-\mathrm{TrFE}-\mathrm{CFE})$ with a composition of $62.3 / 29.9 / 7.8 \mathrm{~mol}$. \%, BNNSs refers to Boron nitride nanosheets (9 vol. \%), and BST67 refers to $\mathrm{Ba}_{0.67} \mathrm{Sr}_{0.33} \mathrm{TiO}_{3}(8$ vol. \%).

${ }^{\mathrm{f}} \mathrm{P}(\mathrm{VDF}-\mathrm{TrFE}) 52 / 48 \mathrm{~mol} \% \%$ and $\mathrm{Ba}_{0.75} \mathrm{Sr}_{0.25} \mathrm{TiO}_{3}(10$ vol. \%).

${ }^{\mathrm{g}}$ Terpolymer refers to P(VDF-TrFE-CFE) (59.4/33.4/7.2 mol. \%) and PMN-PT refers to 0.9PMN-0.1PT nanoparticles with the content of 37.5 wt. \%.

${ }^{\mathrm{h}}$ (VDF-TrFE-CFE)/P(VDF-TrFE) 90/10 wt. \% blend and graphene (1.0 wt. \%). In the method column, SC, SThM, TC, DSC, and IR indicate specific calorimeters, scanning thermal microscopy, thermocouple, scanning thermal microscopy and infra-red camera.

thickness of few micrometers or bulk, including ceramics and single crystals. It would either require the device to be engineered with a thinner substrate, a thermal barrier, and a greater film volume fraction or demand technological achievements to get precise thermal measurements (as in pyroelectric detector devices). More importantly, reasonable calibration has always to be considered to compensate for unavoidable thermal losses during the measurements.

\section{A. Thermocouple and thermometer}

A thermocouple (based on thermoelectric effect) or thermometer (based on various principles) is a simple device to determine the temperature and electrocaloric temperature change. Usually, it is attached on a bulk sample using thermally conducting paste in order to meet good thermal contact between the sample and the thermocouple or thermometer. ${ }^{26,59,60,62,128,138}$ Otherwise, thermal losses may lead to the measurements conducted in non-adiabatic conditions. ${ }^{105}$ The thermocouple or thermometer by itself cannot be used to provide reliable determination of the electrocaloric effect in thin films and is sensitively influenced by the surroundings. However, it can be integrated into a calorimeter system (i.e., high-resolution calorimeter ${ }^{11,58,124,139}$ and scanning thermal microscope ${ }^{104,138}$ ) as one of the key elements to achieve highresolution probe of the electrocaloric effect even in thin films.

\section{B. Differential Scanning Calorimetry (DSC)}

Modified DSC can provide a good and precise characterization of electrocaloric heat of samples in quasi-isothermal conditions. Different from the standard mode measuring zero-field heat capacity, modified DSC evaluates the heat flow $d Q / d E$ by applying an electric field at a controlled temperature. ${ }^{26,43,57,84,129,130,132}$ The electrocaloric heat can be then obtained by $Q=\int_{0}^{E}\left(d Q / d E^{\prime}\right) d E^{\prime}$, which corresponds to the area of the exothermic (endothermic) peak. Therefore, the entropy change can be determined by $\Delta S=Q / T$. To obtain the temperature dependence of heat flow, this procedure is repeated at different measurement temperatures from cooling or heating. Due to the thermal conduction of the apparatus, a long waiting period is usually required to stabilize the temperature before the electrocaloric measurement starts. Therefore, this approach is a slow measurement. The thermal losses from imperfect thermal contact between a sample and DSC sensors should be taken into account. ${ }^{43,57,84}$ In addition, a quasi-direct method by measuring the heat flow $d Q / d T$ at fixed finite field was also reported. ${ }^{26}$ Further information about various DSC setups can be found elsewhere (Refs. 26, 43, 57, 84, 129, 130, and 132 and references therein].

As long as the temperature dependence of entropy change is given by the DSC method, the temperature change is often calculated by $\Delta T=T \Delta S / C(0)$ where a constant zero-field and temperature-independent $C(0)$ are used (Refs. $26,43,57,84,129,130$, and 132 and references therein]. As we analyzed in Section II F, this inappropriate assumption is not supported by recent experimental and theoretical evidence. ${ }^{24,27,70,87,97-100}$ Indeed, a very similar issue was already discussed comprehensively in the magnetocaloric community. For instance, Pecharsky and Gschneidner addressed in detail ${ }^{140}$ that the unrealistic assumption (that 


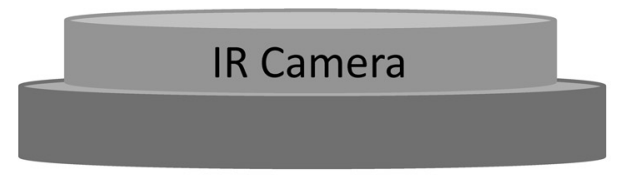

(a)

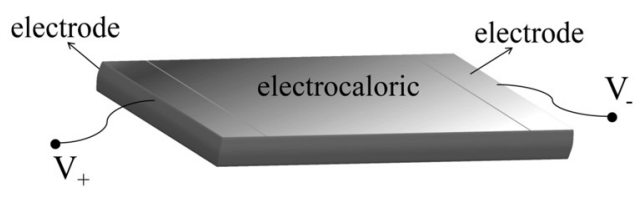

(c)

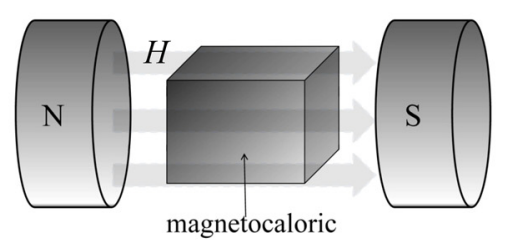

(b)

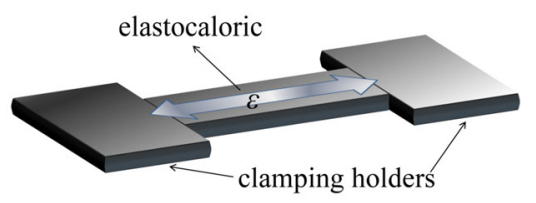

(d)

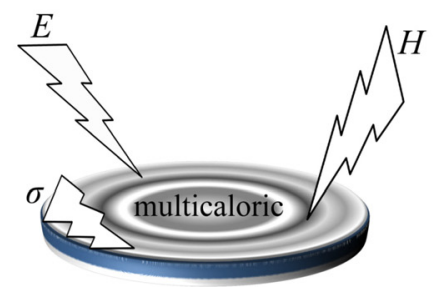

FIG. 10. Schematics of IR measurement set-up for direct image of (a) electrocaloric effect, (b) elastocaloric effect ( $\varepsilon$ is strain and the arrow indicates its direction), (c) magnetocaloric effect ( $H$ refers to magnetic field from the magnets), and (d) multicaloric effect ( $\sigma$ indicates uniaxial stress), respectively. heat capacity is magnetic-field independent) may fail to yield a reasonable approximation for magnetocaloric temperature change. Moreover, they suggested an approach by combining heat capacity and magnetization data to extract $\Delta T$. And even for obtaining the entropy change by integrating the measured heat from DSC, the deleterious effects of intrinsically inaccurate heat capacity should also be eliminated or minimized carefully especially for first-order transitions. ${ }^{141}$ One practical route to ensure reasonable and useful data obtained by DSC is to compare it with other direct measurements or from indirect data based on proper isothermal curves. This demands the modification of the application mode of DSC further to determine the field-dependence of heat capacity or combine other techniques available.

\section{Specifically designed calorimeters}

A specific calorimeter sensor was developed by Zhang's group. ${ }^{124}$ The operational principle is to compare the electrocaloric heat originated from samples with the heat generated by a reference resistor. The reference resistor heater attached to the samples with the same integrated areas produces a Joule heat which can be detected by IR sensor or heat flux sensor. For instance, the voltage signal peaks, detected by the heat flux sensor and then amplified by the low-noise preamplifier, can be observed by the oscilloscope (Refs. 134 and 136 and references therein]. The integrated areas of the peaks correspond to the amount of heat released or absorbed by the reference resistor (Refs. 134 and 136 and references therein]. When the electrocaloric effect is induced in the samples, the electrocaloric heat released or absorbed is then calibrated by this reference resistor and $\Delta T$ can be finally obtained through simple calculations. This technique is useful for bulk samples and thick films $(>1 \mu \mathrm{m})$.

Another specific calorimeter called high-resolution calorimeter was recently developed by Kutnjak et al. to measure directly the electrocaloric effect. ${ }^{11,27,58,70,124,139}$ Highresolution calorimeter was first used to determine the fielddependence of latent heat to study the phase transitions in relaxors. ${ }^{99}$ The latent heat can be extracted by comparing results of the ac mode and the relaxation mode. 1,27,99,100,142 $^{1}$ Moreover, the modified calorimeter is shown to be useful not only in probing the electrocaloric response for bulk including single crystals and ceramics $11,27,41,42,58,70,139$ but also for thin films. ${ }^{124}$ The readers are recommended for further details to Refs. 1 and 11 .

In addition to these two specific calorimeters, there are other examples available according to the literature. For instance, laser-based calorimeter using temperaturedependent reflectance measurements of the top metal (additionally sputtered on the top electrode of the thin films) was used to characterize the electrocaloric effect directly in nanometer-scale thin films. ${ }^{125}$ The experimental results revealed an invisible contribution arising from elastocaloric effect related to substrate clamping through the piezoelectric coefficient of thin films, which is not necessarily taken into account into Equation (1) (see Ref. 125). In other words, Equation (1) using the polarization as the order parameter under proper mechanical boundary conditions ${ }^{24,25}$ may fail to describe the electrocaloric effect. Finally, a homemade calorimeter using a microfabricated resistance thermometer (integrated onto the substrate) was developed by Jia and Ju to provide direct measurement on electrocaloric effect in organic thick films. ${ }^{28}$

\section{Infra-red camera}

Compact and fast IR cameras (see the experimental setup in Figure 10) can provide an accurate and sensitive imaging of caloric effects both temporally and spatially. $^{132,133,138,143-149}$ Typically, the camera consists of an arrangement of lenses with a digital detector semiconductor (i.e., InSb) having a broadband spectral sensitivity of several micrometers. Thermal images captured by the camera usually have an effective spatial resolution of several micrometers with a thermal sensitivity of about $25 \mathrm{mK}$. The IR camera is calibrated with black-body calibration by the provider. For caloric measurements, the frame frequency 
describing the capability of the camera to capture thermal change is one of the key parameters, since it is correlated to the measurement conditions. For instance, higher frequencies (i.e., $100 \mathrm{~Hz}$ ) can ensure the adiabatic conditions in most of the measurements on bulk materials. ${ }^{132,133,138,143-149}$ In this case, the camera is sensitive enough to capture thermal change in each pixel in near adiabatic conditions.

To ensure the high accuracy in temperature measurements, it is usually required that the surface of samples is flat since the surface emissivity is strongly related to its roughness. In the case of a rough surface, the corresponding thermal image recorded by the camera will represent a false profile of inhomogeneity in temperature before the caloric effect is driven by an external stimulus. ${ }^{138}$ This can be overcome by measuring the intensity of the thermal radiation at two adjacent temperatures and interpolating linearly but at expense of the high accuracy temperature measurements. ${ }^{138}$ This can be also achieved by manually adjusting the emissivity of different selected regions without compromising the resolution of camera, ${ }^{149}$ which corresponds to setting the temperature of the sample to a specific measurement temperature.

As mentioned above, an IR camera has been frequently used to measure the magnetocaloric, elastocaloric, and electrocaloric effects. ${ }^{132,133,138,143-149}$ A non-contact IR camera is compact and fast compared with the other techniques, i.e., DSC, and its use is rather simple. More importantly, an IR camera can directly image spatial caloric response, which is the main advantage over other techniques such as DSC, thermocouple, and other specifically designed calorimeters. Recent studies specifically demonstrated that IR imaging as a function of real time and magnitude of stimulus can provide reliable data for caloric response with good precision. ${ }^{132,138,144,146}$ For instance, in order to ensure the reliability of this technique, data obtained by an IR camera are compared with those from thermocouple ${ }^{138,144,146}$ and DSC measurements ${ }^{132}$ and were found to be consistent. An IR camera is very sensitive to the surroundings, the people who are carrying out this kind of measurement not mentioning the inevitable temperature variation in a day. This makes temperature dependence of measurement difficult since the temperature fluctuations can directly contribute to the thermal images of the camera if no thermal shield is arranged. In addition, IR measurement is usually limited in a temperature window [about $0-100^{\circ} \mathrm{C}$ ] near room temperature.

In addition to temporally resolved measurement, spatial thermal images captured by IR cameras are an ideal noncontact tool to explore the distribution of temperature profiles subjected to external stimuli such as uniaxial stress. ${ }^{144,147,148,150}$ In this case, the thermal response is correlated with structural degrees of freedom of materials, which can be very useful to study the microstructure dynamics such as nucleation. ${ }^{144,148}$ Spatially resolved measurements have been conducted to study the local inhomogeneities of elastocaloric and magnetocaloric responses. ${ }^{143,144,147,148}$ However, similar studies on electrocaloric effect are rarely reported. In turn, electrocaloric effect is always characterized by an isotropic $\Delta T$ with a homogeneous distribution along the sample in the literature except for very few studies. ${ }^{138,151,152}$ For instance, it was reported that uniaxial $\mathrm{Sr}_{0.75} \mathrm{Ba}_{0.25} \mathrm{Nb}_{2} \mathrm{O}_{6}$ and $\mathrm{Pb}\left(\mathrm{Mg}_{1 / 3} \mathrm{Nb}_{2 / 3}\right) \mathrm{O}_{3}-\mathrm{PbTiO}_{3}$ relaxor single crystals display anisotropy of electrocaloric cooling (about $0.20-0.25 \mathrm{~K}$ ) due to strong polarization anisotropy ${ }^{151}$ and different crystal orientations. ${ }^{152}$ In addition, extrinsic variation of electrocaloric effect (approximately $0.2 \mathrm{~K}$ ) due to specific geometry in multilayer capacitors was observed between the metallic terminals and electrocaloric active ceramic regions in multilayer capacitors. ${ }^{138}$ This underlines that spatially distributed electrocaloric effects should exist over the multilayer capacitors in contrast to theoretical assumptions. ${ }^{153,154}$ Moreover, using IR camera, Liu et al. revealed the existence of a significant variation of electrocaloric effect up to $0.285 \mathrm{~K}$ between the central region and the region near the terminals. ${ }^{149}$ This result corresponds to about $50 \%$ of the average value in the sample, which is larger than the previous ones on anisotropy and inhomogeneity of electrocaloric cooling. ${ }^{138,151,152}$

Let us briefly recall that current interest in design of electrocaloric prototypes is mainly based on multilayer capacitors. The multilayer capacitors consist of around 200 ceramic layers each of thickness about $10 \mu \mathrm{m}$ separated by interdigitated inner electrodes with a thickness of several $\mu \mathrm{m}$. The electrocaloric effect in multilayer capacitors was already reported. ${ }^{15,127}$ But it is only recently that the promise of multilayer capacitors in practical electrocaloric refrigeration was addressed ${ }^{22,128,129,153}$ and was regarded as one of the main breakthroughs in this field. ${ }^{3}$ Using multilayer capacitors is a successful compromise allowing high breakdown field and relative large electrocaloric active volume. Moreover, electrocaloric refrigerator prototypes based on multilayer capacitors were designed, confirming the potential of multilayer capacitors for real cooling applications. ${ }^{19,20,22,155}$ In this case, the IR camera is a powerful tool to detect the dynamic performances of multilayer capacitors, i.e., the electrocaloric response under external fields with different temporal shapes and frequencies, ${ }^{156}$ the contributions of Joule heating, ${ }^{30,157}$ as well as the electrocaloric fatigue properties under cyclic operating. These unexplored data are extremely desirable to the design of electrocaloric prototypes.

Note that understandings of the heat flow behavior in specific caloric prototype devices are crucial to optimize the refrigeration efficiency. ${ }^{94}$ For instance, control of heat flux direction in active magnetic regeneration in an effective manner can be very useful to enhance the operating frequency and thus the power density of the device. ${ }^{158}$ Direct thermal mapping captured by IR camera allows analyzing qualitatively the dynamic heat flux in one specific prototype device according to distributions of adiabatic temperature change. For instance, the electrocaloric heat flow in multilayer capacitors is found to be mainly bidirectional but is transferring inhomogeneously between the central ceramic layers and the terminals. ${ }^{149}$ To be specific, the electrocaloric heat is mainly transferred in a path along interdigitated inner electrodes away from (towards) the terminals and it is weakly dissipated in the direction along the terminals in a complex manner. ${ }^{149}$ Moreover, quantitative analysis about electrocaloric heat flux in multilayer capacitors can be achieved by combining both spatially and temporally 
resolved measurements. For instance, a sustained cooling power of $0.037 \mathrm{~kW} / \mathrm{m}^{2}$ is experimentally obtained based on spatially resolved imaging of temperature gradient. ${ }^{149}$ In addition, we note that IR camera is also capable of measuring the multicaloric effect (see Figure 10(d)) driven by either single stimulus ${ }^{159}$ or multiple stimuli (applied/removed simultaneously or sequentially). ${ }^{8}$

\section{E. Scanning thermal microscopy}

Based on scanning probe microscopy, scanning thermal microscopy is a useful technique permitting the thermal probing of the submicron heat transfer behavior of materials and devices. ${ }^{160,161}$ Recently, Kar-Narayan et al. introduced this approach for directly measuring the electrocaloric effect in multilayer capacitors ${ }^{138}$ and relaxor thin films. ${ }^{104}$ In the measurement set-up, the atomic force microscope tip is not in contact with the samples. A small resistance thermometer deposited in the tip is used to detect the temperature change through tip-sample heat transfer when the electric field is applied and removed. It was also found that the measured temperature change and its shape with respect to time strongly depend on the distance between tip and are specifically measured region in both studies. ${ }^{104,138}$ The measurement can be conducted quickly with a resolution of temperature being $80 \mathrm{mK} .{ }^{138}$ Scanning of the tip can therefore provide the spatial resolution of electrocaloric response of the sample, like the IR camera. Near adiabatic conditions can be met when active electrocaloric active volume is large, e.g., in multilayer capacitors. ${ }^{138}$ However, measurements in thin films (13 and $38 \mu \mathrm{m}$ ) suffer from non-adiabatic conditions due to the rapid heat exchange between the film and underneath substrate. ${ }^{104}$ Obviously, further works exploiting this technique are needed in future.

\section{PERSPECTIVES}

The application of electrocalorics is not limited to onchip cooling or other nano-electronics embodiments as proposed originally in thin films. ${ }^{16,17}$ Electrocaloric cooling will be probably limited to situations with small temperature ranges and modest cooling rates and loads. However, their other attributes such as low power, inexpensive components, and modest size may be advantageous. For instance, there are also other products where constant but modest temperatures are required: transplant organs for surgery must be transported at long distances and neither frozen nor allowed to warm but kept at a constant temperature, e.g., $4{ }^{\circ} \mathrm{C}$. Other applications exist where size, weight, and power consumption are paramount, such as refrigerant-free devices in commercial satellites. In general, it is useful to keep in mind that medical applications and aerospace or aircraft devices involve different priorities for performance parameters.

There is a yet unexploited commercial application for ferroelectrics near quantum critical points (i.e., for $T_{\mathrm{C}} \rightarrow$ $0 \mathrm{~K})$. These include tris-sarcosine calcium bromide (TSSB), $\mathrm{Ba}-, \mathrm{Sr}-$, or $\mathrm{Pb}$-hexaferrites $\left(\mathrm{MFe}_{12} \mathrm{O}_{19}\right)$, and others. Readers should be aware that the electrocaloric and magnetocaloric effects are useful not just for near-room-temperature devices, but also for cryogenics (especially the later has been commercialized). There is a long history led by Lawless (Ref. 127 and others) of using ferroelectric anomalies in perovskite oxides as precision thermometers for $T$ in the $\mathrm{mK}$ regime. Of course, electrocalorics have an advantage in not requiring magnets. In the future, a severe worldwide shortage of $\mathrm{He}$ is expected, with consequent increase in price, and we note that cryogen-free cryostats are already commercially available: e.g., Cambridge Cryogenics. www.cambridgecryogenics.com (the present authors have no connection with this company). Their systems are fitted with coaxial wires to the $\mathrm{mK}$ temperature range and magnets ideal for ferroelectric and multiferroic measurements. Tuning parameters include temperature, magnetic field, electric field, field angle (computer controlled rotator), and pressure. Extension to multiferroic coolers is likely in the near future, with temperature ranges down to the $\mathrm{mK}$ regime. On 16 September 2016, the US Navy is launching a mini-satellite with a ferroelectric memory for "cold airless space." Further satellite applications are eminent in China.

In addition, since all present electrocalorics should generally work in the relatively small voltage regime, extension to very high fields may develop soon, e.g., in multilayer capacitors. Oxide perovskites can withstand breakdown fields up to 1 or $2 \mathrm{GV} / \mathrm{m} .{ }^{162}$ When very large fields are applied, reversible wrinkling can occur, adding additional entropy. ${ }^{163}$ However, if fields are too high, irreversible folding occurs, for example, the Helfrich-Hursault lamellar instability in ferroelectric $C^{*}$ smectic liquid crystals or the Ramberg-Osgood instability in other materials. ${ }^{164,165}$

Finally, careful examination should be made for electrocaloric properties of voltage-driven Mott transitions, such as $\mathrm{NdNiO}_{3}$. Here, there is a significant entropy change with either voltage or temperature. Interestingly, a remarkable electrocaloric effect (about $-3.8 \mathrm{~K}$ under a voltage change of 0-3 V) was observed in bulk $\mathrm{VO}_{2}$ due to electric-field induced metal-insulator phase transition. ${ }^{166}$ Note that in such materials, thermoelectric effect, i.e., variation of temperature through the electrical current, can also contribute to the caloric response. On the other hand, we have mainly concentrated on reviewing the inorganic electrocalorics, while there is no denying that polymeric materials such as copolymers of PVDF and trifluoroethylene are also very promising for developing electrocaloric prototype devices due to their large electrocaloric response and flexible nature. ${ }^{17,55,123}$ Review on organic electrocalorics can be found in Refs. 1, 3, 4, and 6. It is difficult to make a conclusion on which material is the better especially between inorganic ceramics and organic polymers. ${ }^{167}$ In this regard, the compromise emerging as composite materials by combining these two compounds together may be promising. The concept of using nanocomposites was first proposed to obtain enhanced electrocaloric cooling. ${ }^{168}$ Moreover, a colossal electrocaloric effect was reported very recently in ferroelectric polymer nanocomposites by taking advantage of the significantly enhanced breakdown field ${ }^{134,136,169}$ due to the presence of the boron nitride nanosheets (BNNSs). ${ }^{170}$ The electrocaloric properties of nanocomposites even surpass those of the recently reported giant magnetocalorics, ${ }^{12}$ which demonstrates the interest and potential role of electrocaloric effect in 
next-generation refrigeration. For instance, composites combining relaxor terpolymer $\mathrm{P}(\mathrm{VDF}-\mathrm{TrFE}-\mathrm{CFE}), \mathrm{Ba}_{0.67}$ $\mathrm{Sr}_{0.33} \mathrm{TiO}_{3}$ ceramics, and BNNSs show a huge electrocaloric temperature change of over $50 \mathrm{~K}$ at room temperature under a ultrahigh electric field of $2500 \mathrm{kV} / \mathrm{cm},{ }^{134}$ while relaxor composites based on P(VDF-TrFE-CFE) and PMN-PT ${ }^{136}$ are also demonstrated to be comparable to this performance (see Table I). Testifying the giant electrocaloric effect in other nanostructures such as nanoparticles, ${ }^{106,111,171}$ nanotubes, ${ }^{172}$ and nanowires ${ }^{173}$ is highly desired since recent experimental results demonstrated that electrocaloric effect in polymer nanocomposites can be tailored by the morphology of the ferroelectric nanofillers. ${ }^{169}$ As a result, the idea of using nanowires for towards wearable cooling applications has been achieved in highly bendable and stretchable $\mathrm{Ba}_{0.67}$ $\mathrm{Sr}_{0.33} \mathrm{TiO}_{3}$ nanowires arrays. ${ }^{174}$ In this case, experimental results show that sizable electrocaloric response could remain in these highly flexible nanowire arrays under a safe voltage $(<36 \mathrm{~V})$, mechanical stretching (25\%) up to 10000 cycles. ${ }^{174}$ Interestingly, combining electrocalorics with magnetocalorics, i.e., in the form of multiferroic heterostructure, may be useful to organize a multicaloric cycle and design of giant electric-field driven caloric effect, which was demonstrated very recently in $\mathrm{FeRh} / \mathrm{BaTiO}_{3}$ heterostructure. ${ }^{175}$

\section{CLOSING REMARKS}

Critical insights into the indirect approach which is widely used without criticism are summarized here incorporating recent theoretical and experimental advances, which are dedicated to prolong any unnecessary misunderstandings or feuds. The role of field dependence of heat capacity is addressed although no definitive answer to a reliable approach to select proper $P(E)$ loops is provided. Generally, it is yet required to take into account the important effects such as ferroelectric switching, kinetics such as measurement frequency, electrical hysteresis losses between application and removal of electric field, thermal hysteresis associated with first-order phase transition between cooling and heating processes, and so on. This obviously demands further studies. Nevertheless, indirect measurement based on the Maxwell relations could provide general trends to analyze the behavior of electrocaloric responses and might be useful to compare different results obtained from the same class of materials. It may be still useful acting as an approximate tool to estimate the electrocaloric effect at current research stage.

Ultimately, direct measurements of heat and temperature change are highly desired for indisputable proof of electrocaloric effect at this stage. In particular, we address the promise of IR cameras in directly imaging electrocaloric effect from material characterization to design of electrocaloric prototypes. The commercial feature makes this technique competitive for providing a reliable experimental data, at least comparable from a research group to another. Finally, we hope that our review can be found useful in some aspects and read as an inspiration for the fast growing interest in the field of electrocalorics.

\section{ACKNOWLEDGMENTS}

Y.L. and B.D. acknowledge the China Scholarship Council (CSC) for funding Y.L.'s stay in France and a public grant overseen by the French National Research Agency (ANR) as part of the "Investissements d'Avenir" program (Reference: ANR-10-LABX-0035, Labex NanoSaclay). The authors thank L. Bellaiche for fruitful discussions and W. P. Geng and E. Defay for experimental collaborations.

${ }^{1}$ T. Correia and Q. Zhang, Electrocaloric Materials: New Generation of Cooler (Springer Verlag, Berlin, Heidelberg, London, UK, 2014).

${ }^{2}$ J. F. Scott, Science 315, 954 (2007).

${ }^{3}$ J. F. Scott, Annu. Rev. Mater. Res. 41, 229 (2011).

${ }^{4}$ S. G. Lu and Q. Zhang, Adv. Mater. 21, 1983 (2009).

${ }^{5}$ M. Valant, Prog. Mater. Sci. 57, 980 (2012).

${ }^{6}$ X. Y. Li, S.-G. Lu, X.-Z. Chen, H. M. Gu, X.-S. Qian, and Q. M. Zhang, J. Mater. Chem. C 1, 23 (2013).

${ }^{7}$ S. Pamir Alpay, J. Mantese, S. Trolier-McKinstry, Q. M. Zhang, and R. W. Whatmore, MRS Bull. 39, 1099 (2014).

${ }^{8}$ X. Moya, S. Kar-Narayan, and N. D. Mathur, Nat. Mater. 13, 439 (2014).

${ }^{9}$ I. Takeuchi and K. Sandeman, Phys. Today 68(12), 48 (2015).

${ }^{10}$ S. Crossley, N. D. Mathur, and X. Moya, AIP Adv. 5, 067153 (2015).

${ }^{11}$ Z. Kutnjak, B. Rožič, and R. Pirc, Wiley Encyclopedia of Electrical Electronics Engineering (John Wiley \& Sons, 2015), 1-19.

${ }^{12}$ A. Kitanovski, U. Plaznik, U. Tomc, and A. Poredoš, Int. J. Refrig. 57, 288 (2015).

${ }^{13}$ P. Kobeko and J. Kurtschatov, Z. Phys. 66, 192 (1930).

${ }^{14}$ B. A. Tuttle and D. A. Payne, Ferroelectrics 37, 603 (1981).

${ }^{15}$ L. Shebanovs, K. Borman, W. N. Lawless, and A. Kalvane, Ferroelectrics 273, 137 (2002).

${ }^{16}$ A. S. Mischenko, Q. Zhang, J. F. Scott, R. W. Whatmore, and N. D. Mathur, Science 311, 1270 (2006).

${ }^{17}$ B. Neese, B. Chu, S. G. Lu, Y. Wang, E. Furman, and Q. M. Zhang, Science 321, 821 (2008).

${ }^{18}$ A. Kitanovski, J. Tušek, U. Tomc, U. Plaznik, M. Ožbolt, and A. Poredoš, Magnetocaloric Energy Conversion: From Theory to Applications (Springer International Publishing Switzerland, 2015).

${ }^{19}$ Y. B. Jia and Y. Sungtaek Ju, Appl. Phys. Lett. 100, 242901 (2012).

${ }^{20}$ H. Gu, X. S. Qian, X. Li, B. Craven, W. Zhu, A. Cheng, S. C. Yao, and Q. M. Zhang, Appl. Phys. Lett. 102, 122904 (2013).

${ }^{21}$ H. Gu, X. S. Qian, H. J. Ye, and Q. M. Zhang, Appl. Phys. Lett. 105, 162905 (2014).

${ }^{22}$ R. I. Epstein and K. J. Malloy, J. Appl. Phys. 106, 064509 (2009).

${ }^{23}$ X. Y. Li, H. M. Gu, X. S. Qian, and Q. M. Zhang, "Thermal and thermomechanical phenomena in electronic systems (ITherm)," in 13th IEEE Intersociety Conference (30 May 2012-1 June 2012), pp. 934-937.

${ }^{24}$ G. Akcay, S. P. Alpay, J. G. A. Rossetti, and J. F. Scott, J. Appl. Phys. 103, 024104 (2008).

${ }^{25}$ S. G. Lu, B. Rozic, Q. M. Zhang, Z. Kutnjak, and R. Pirc, Appl. Phys. A 107, 559 (2012).

${ }^{26}$ X. Moya, E. Stern-Taulats, S. Crossley, D. González-Alonso, S. KarNarayan, A. Planes, L. Mañosa, and N. D. Mathur, Adv. Mater. 25, 1360 (2013).

${ }^{27}$ N. Novak, R. Pirc, and Z. Kutnjak, Phys. Rev. B 87, 104102 (2013).

${ }^{28}$ Y. B. Jia and Y. S. Ju, Appl. Phys. Lett. 103, 042903 (2013).

${ }^{29}$ W. P. Geng, Y. Liu, X. J. Meng, L. Bellaiche, J. F. Scott, B. Dkhil, and A. Q. Jiang, Adv. Mater. 27, 3165 (2015).

${ }^{30}$ M. Quintero, P. Gaztañaga, and I. Irurzun, Appl. Phys. Lett. 107, 151901 (2015).

${ }^{31}$ S. Crossley, "Electrocaloric materials and devices," Ph.D. thesis (University of Cambridge, 2013).

${ }^{32}$ J. S. Young, "Indirect measurement of the electrocaloric effect," Ph.D. thesis (University of Cambridge, 2011).

${ }^{33}$ K. M. Rabe, in Antiferroelectricity in Oxides: A Reexamination, in Functional Metal Oxides: New Science and Novel Applications, edited by S. B. Ogale, T. V. Venkatesan, and M. G. Blamire (Wiley-VCH, Weinheim, Germany, 2014), Chap. 7.

${ }^{34}$ J. Parui and S. B. Krupanidhi, Phys. Status Solidi RRL 2, 230 (2008).

${ }^{35}$ X. H. Hao, Z. X. Yue, J. B. Xu, S. L. An, and C.-W. Nan, J. Appl. Phys. 110, 064109 (2011). 
${ }^{36}$ Y. Bai, G.-P. Zheng, and S.-Q. Shi, Mater. Res. Bull. 46, 1866 (2011).

${ }^{37}$ B. Peng, H. Fan, and Q. Zhang, Adv. Funct. Mater. 23, 2987 (2013).

${ }^{38}$ X. J. Jiang, L. H. Luo, B. Y. Wang, W. P. Li, and H. B. Chen, Ceram. Int. 40, 2627 (2014).

${ }^{39}$ Y. Zhao, X. H. Hao, and Q. Zhang, ACS Appl. Mater. Interfaces 6, 11633 (2014)

${ }^{40}$ W. P. Cao, W. L. Li, D. Xu, Y. F. Hou, W. Wang, and W. D. Fei, Ceram. Int. 40, 9273 (2014).

${ }^{41}$ R. Pirc, B. Rožič, J. Koruza, B. Malič, and Z. Kutnjak, Europhys. Lett. 107, 17002 (2014).

${ }^{42}$ R. Pirc, B. Rožič, J. Koruza, G. Cordoyiannis, B. Malič, and Z. Kutnjak, J. Phys.: Condens. Matter 27, 455902 (2015).

${ }^{43}$ F. Le Goupil, J. Bennett, A.-K. Axelsson, M. Valant, A. Berenov, A. J. Bell, T. P. Comyn, and N. McN Alford, Appl. Phys. Lett. 107, 172903 (2015)

${ }^{44}$ K. A. Gschneidner, Jr., V. K. Pecharsky, E. Brück, H. G. M. Duijn, and E. M. Levin, Phys. Rev. Lett. 85, 4190 (2000).

${ }^{45}$ S. A. Nikitin, G. Myalikgulyev, A. M. Tishin, M. P. Annaorazov, K. A. Asatryan, and A. L. Tyurin, Phys. Lett. A 148, 363 (1990).

${ }^{46}$ T. Tohei, H. Wada, and T. Kanomata, J. Appl. Phys. 94, 1800 (2003).

${ }^{47}$ T. Krenke, E. Duman, M. Acet, E. F. Wassermann, X. Moya, L. Mañosa, and A. Planes, Nat. Mater. 4, 450 (2005).

${ }^{48}$ J. Shen, J.-L. Zhao, F.-X. Hu, J.-F. Wu, M.-Q. Gong, Y.-X. Li, J.-R. Sun, and B.-G. Shen, J. Appl. Phys. 107, 09A931 (2010).

${ }^{49}$ A. Midya, S. N. Das, P. Mandal, S. Pandya, and V. Ganesan, Phys. Rev. B 84, 235127 (2011).

${ }^{50}$ H. Zhang, Y. W. Li, E. K. Liu, Y. J. Ke, J. L. Jin, Y. Long, and B.-G. Shen, Sci. Rep. 5, 11929 (2015).

${ }^{51}$ A. Maurya, A. Thamizhavel, P. Bonville, and S. K. Dharar, e-print arXiv:1507.05743.

${ }^{52}$ K. Nishimura, Y. Nakazawa, L. W. Li, and K. Mori, Mater. Trans. 49, 1753 (2008)

${ }^{53}$ A. R. Akbarzadeh, S. Prosandeev, E. J. Walter, A. Al-Barakaty, and L. Bellaiche, Phys. Rev. Lett. 108, 257601 (2012).

${ }^{54}$ S. Lisenkov, B. K. Mani, E. Glazkova, C. W. Miller, and I. Ponomareva, Sci. Rep. 6, 19590 (2016).

${ }^{55}$ S. G. Lu, B. Rožič, Q. M. Zhang, Z. Kutnjak, R. Pirc, M. Lin, X. Li, and L. Gorny, Appl. Phys. Lett. 97, 202901 (2010).

${ }^{56}$ X.-S. Qian, H.-J. Ye, Y.-T. Zhang, H. Gu, X. Li, C. A. Randall, and Q. M. Zhang, Adv. Funct. Mater. 24, 1300 (2014).

${ }^{57}$ M. Sanlialp, V. V. Shvartsman, M. Acosta, B. Dkhil, and D. C. Lupascu, Appl. Phys. Lett. 106, 062901 (2015).

${ }^{58}$ B. Rožič, M. Kosec, H. Uršič, J. Holc, B. Malič, Q. M. Zhang, R. Blinc, R. Pirc, and Z. Kutnjak, J. Appl. Phys. 110, 064118 (2011).

${ }^{59}$ J. Hagberg, A. Uusimäki, and H. Jantunen, Appl. Phys. Lett. 92, 132909 (2008).

${ }^{60}$ L. J. Dunne, M. Valant, A.-K. Axelsson, G. Manos, and N. M. Alford, J. Phys. D: Appl. Phys. 44, 375404 (2011).

${ }^{61}$ M. Valant, L. J. Dunne, A.-K. Axelsson, N. M. Alford, G. Manos, J. Peräntie, J. Hagberg, H. Jantunen, and A. Dabkowski, Phys. Rev. B 81, 214110 (2010).

${ }^{62}$ J. Peräntie, J. Hagberg, A. Uusimäki, and H. Jantunen, Phys. Rev. B 82, 134119 (2010).

${ }^{63}$ Y. P. Shi and A. K. Soh, Acta Mater. 59, 5574 (2011).

${ }^{64}$ R. Pirc, Z. Kutnjak, R. Blinc, and Q. M. Zhang, Appl. Phys. Lett. 98, 021909 (2011).

${ }^{65}$ Z. K. Liu, X. Li, and Q. M. Zhang, Appl. Phys. Lett. 101, 082904 (2012)

${ }^{66}$ Y. Bai, X. Han, and L. J. Qiao, Appl. Phys. Lett. 102, 252904 (2013).

${ }^{67}$ Z. D. Luo, D.-W. Zhang, Y. Liu, D. Zhou, Y. G. Yao, C. Q. Liu, B. Dkhil, X. B. Ren, and X. J. Lou, Appl. Phys. Lett. 105, 102904 (2014).

${ }^{68}$ X. J. Wang, F. Tian, C. Zhao, J. Wu, Y. Liu, B. Dkhil, M. Zhang, Z. Gao, and X. J. Lou, Appl. Phys. Lett. 107, 252905 (2015).

${ }^{69}$ R. Pirc, Z. Kutnjak, R. Blinc, and Q. M. Zhang, J. Appl. Phys. 110, 074113 (2011)

${ }^{70}$ N. Novak, Z. Kutnjak, and R. Pirc, Europhys. Lett. 103, 47001 (2013).

${ }^{71}$ Y. Liu, J. Wei, P.-E. Janolin, I. C. Infante, J. Kreisel, X. J. Lou, and B. Dkhil, Phys. Rev. B 90, 104107 (2014).

${ }^{72}$ Y. Liu, J. Wei, P.-E. Janolin, I. C. Infante, X. J. Lou, and B. Dkhil, Appl. Phys. Lett. 104, 162904 (2014).

${ }^{73}$ E. Mikhaleva, I. Flerov, M. Gorev, M. Molokeev, A. Cherepakhin, A. Kartashev, N. Mikhashenok, and K. Sablina, Phys. Solid State 54, 1832 (2012).

${ }^{74}$ S. Lisenkov and I. Ponomareva, Phys. Rev. B 86, 104103 (2012).
${ }^{75}$ S. Lisenkov, B. K. Mani, C.-M. Chang, J. Almand, and I. Ponomareva, Phys. Rev. B 87, 224101 (2013).

${ }^{76}$ Y. Liu, I. C. Infante, X. J. Lou, L. Bellaiche, J. F. Scott, and B. Dkhil, Adv. Mater. 26, 6132 (2014).

${ }^{77}$ Y. Liu, I. C. Infante, X. J. Lou, D. C. Lupascu, and B. Dkhil, Appl. Phys. Lett. 104, 012907 (2014).

${ }^{78}$ Y. Liu, J. Wei, X. J. Lou, L. Bellaiche, J. F. Scott, and B. Dkhil, Appl. Phys. Lett. 106, 032901 (2015).

${ }^{79}$ A. Chauhan, S. Patel, and R. Vaish, Appl. Phys. Lett. 106, 172901 (2015).

${ }^{80}$ A. Chauhan, S. Patel, and R. Vaish, Acta Mater. 89, 384 (2015).

${ }^{81}$ P. Lloveras, E. Stern-Taulats, M. Barrio, J.-L. Tamarit, S. Crossley, W. Li, V. Pomjakushin, A. Planes, L. Mañosa, N. D. Mathur, and X. Moya, Nat. Commun. 6, 8801 (2015).

${ }^{82}$ S. Patel, A. Chauhan, R. Vaish, and P. Thomas, Appl. Phys. Lett. 108, 072903 (2016).

${ }^{83}$ S. Prosandeev, I. Ponomareva, and L. Bellaiche, Phys. Rev. B 78, 052103 (2008).

${ }^{84}$ F. Le Goupil, A. Berenov, A.-K. Axelsson, and M. Valant, J. Appl. Phys. 111, 124109 (2012).

${ }^{85}$ A. K. Axelsson, F. L. Goupil, L. J. Dunne, G. Manos, M. Valant, and N. McN Alford, Appl. Phys. Lett. 102, 102902 (2013).

${ }^{86}$ J. Íñiguez, M. Stengel, S. Prosandeev, and L. Bellaiche, Phys. Rev. B 90, 220103(R) (2014).

${ }^{87}$ M. Marathe, A. Grünebohm, T. Nishimatsu, P. Entel, and C. Ederer, Phys. Rev. B 93, 054110 (2016).

${ }^{88}$ T. M. Correia, J. S. Young, R. W. Whatmore, J. F. Scott, N. D. Mathur, and Q. Zhang, Appl. Phys. Lett. 95, 182904 (2009).

${ }^{89}$ V. Provenzano, A. J. Shapiro, and R. D. Shull, Nature (London) 429, 853-857 (2004).

${ }^{90}$ V. Bassoa, C. P. Sasso, G. Bertotti, and M. LoBue, Int. J. Refrig. 29, 1358 (2006).

${ }^{91}$ J. Lyubina, R. Schäfer, N. Martin, L. Schultz, and O. Gutfleisch, Adv. Mater. 22, 3735 (2010).

${ }^{92}$ J. Liu, T. Gottschall, K. P. Skokov, J. D. Moore, and O. Gutfleisch, Nat. Mater. 11, 620 (2012).

${ }^{93}$ F. Guillou, G. Porcari, H. Yibole, N. Dijk, and E. Brück, Adv. Mater. 26, 2671 (2014).

${ }^{94}$ L. von Moos, K. K. Nielsen, K. Engelbrecht, and C. R. H. Bahl, Int. J. Refrig. 37, 303 (2014).

${ }^{95}$ I. Ponomareva and S. Lisenkov, Phys. Rev. Lett. 108, 167604 (2012).

${ }^{96}$ M. C. Rose and R. E. Cohen, Phys. Rev. Lett. 109, 187604 (2012).

${ }^{97}$ A. Kukreti, A. Kumar, and U. C. Naithani, Indian J. Pure Appl. Phys. 47, 43 (2009).

${ }^{98}$ W. Reese, Phys. Rev. 181, 905 (1969).

${ }^{99}$ Z. Kutnjak, J. Petzelt, and R. Blinc, Nature (London) 441, 956 (2006).

${ }^{100}$ N. Novak, R. Pirc, M. Wencka, and Z. Kutnjak, Phys. Rev. Lett. 109, 037601 (2012).

${ }^{101}$ M. Dawber, K. M. Rabe, and J. F. Scott, Rev. Mod. Phys. 77, 1083 (2005).

${ }^{102}$ S. E. Rowley, M. Hadjimichael, M. N. Ali, Y. C. Durmaz, J. C. Lashley, R. J. Cava, and J. F. Scott, J. Phys.: Condens. Matter 27, 395901 (2015).

${ }^{103}$ K. Ding, Y. Bai, X. Han, W. J. Zhang, and L. J. Qiao, Key Eng. Mater. 492, 164 (2012).

${ }^{104}$ S. Crossley, T. Usui, B. Nair, S. Kar-Narayan, X. Moya, S. Hirose, A. Ando, and N. D. Mathur, Appl. Phys. Lett. 108, 032902 (2016).

${ }^{105}$ J. F. Wang, T. Q. Yang, K. Wei, and X. Yao, Appl. Phys. Lett. 102, 152907 (2013).

${ }^{106}$ Y. K. Zeng, B. Li, J. B. Wang, X. L. Zhong, W. Wang, F. Wang, and Y. C. Zhou, RSC Adv. 4, 30211 (2014).

${ }^{107}$ B. Li, J. B. Wang, X. L. Zhong, F. Wang, Y. K. Zeng, and Y. C. Zhou, Europhys. Lett. 102, 47004 (2013).

${ }^{108}$ J. Wang, M. Liu, Y. J. Zhang, T. Shimada, S.-Q. Shi, and T. Kitamura, J. Appl. Phys. 115, 164102 (2014).

${ }^{109}$ G. Catalan, J. Seidel, R. Ramesh, and J. F. Scott, Rev. Mod. Phys. 84, 119 (2012).

${ }^{110}$ J. Karthik and L. W. Martin, Appl. Phys. Lett. 99, 032904 (2011).

${ }^{111}$ B. Li, J. B. Wang, X. L. Zhong, F. Wang, B. L. Liu, and Y. C. Zhou, J. Nanopart. Res. 15, 1427 (2013).

${ }^{112}$ Y.-B. Ma, K. Albe, and B.-X. Xu, Phys. Rev. B 91, 184108 (2015).

${ }^{113}$ J. A. Brug and W. P. Wolf, J. Appl. Phys. 57, 4685 (1985); 57, 4695 (1985).

${ }^{114}$ R. Caballero-Flores, V. Franco, A. Conde, and L. F. Kiss, J. Appl. Phys. 105, 07A919 (2009).

${ }^{115}$ C. R. H. Bahl and K. K. Nielsen, J. Appl. Phys. 105, 013916 (2009). 
${ }^{116}$ K. K. Nielsen, A. Smith, C. R. H. Bahl, and U. L. Olsen, J. Appl. Phys. 112, 094905 (2012).

${ }^{117}$ C. Romero-Muñiz, J. J. Ipus, J. S. Blázquez, V. Franco, and A. Conde, Appl. Phys. Lett. 104, 252405 (2014).

${ }^{118}$ J. Junquera and P. Ghosez, Nature 422, 506 (2003).

${ }^{119}$ Y. Liu, X. Peng, X. Lou, and H. Zhou, Appl. Phys. Lett. 100, 192902 (2012).

${ }^{120}$ Y. Liu, X. J. Lou, M. Bibes, and B. Dkhil, Phys. Rev. B 88, 024106 (2013)

${ }^{121}$ Y. Liu, I. C. Infante, X. J. Lou, and B. Dkhil, Appl. Phys. Lett. 104, 082901 (2014)

${ }^{122}$ E. Glazkova, C.-M. Chang, S. Lisenkov, B. K. Mani, and I. Ponomareva, Phys. Rev. B 92, 064101 (2015).

${ }^{123}$ P. Liu, J. L. Wang, X. J. Meng, J. Yang, B. Dkhil, and J. H. Chu, New J. Phys. 12, 023035 (2010).

${ }^{124}$ S. G. Lu, B. Rožič, Q. M. Zhang, Z. Kutnjak, X. Y. Li, E. Furman, L. J. Gorny, M. R. Lin, B. Malič, M. Kosec, R. Blinc, and R. Pirc, Appl. Phys. Lett. 97, 162904 (2010).

${ }^{125}$ T. Tong, J. Karthik, R. V. K. Mangalam, L. W. Martin, and D. G. Cahill, Phys. Rev. B 90, 094116 (2014).

${ }^{126}$ H.-J. Ye, X.-S. Qian, D.-Y. Jeong, S. J. Zhang, Y. Zhou, W.-Z. Shao, L. Zhen, and Q. M. Zhang, Appl. Phys. Lett. 105, 152908 (2014).

${ }^{127}$ W. N. Lawless and C. F. Clark, Phys. Rev. B 36, 459 (1987).

${ }^{128}$ S. Kar-Narayana and N. D. Mathur, J. Phys. D: Appl. Phys. 43, 032002 (2010)

${ }^{129}$ Y. Bai, G. P. Zheng, and S. Q. Shi, Appl. Phys. Lett. 96, 192902 (2010).

${ }^{130}$ Y. Bai, G. P. Zheng, K. Ding, L. Qiao, S. Q. Shi, and D. Guo, J. Appl. Phys. 110, 094103 (2011).

${ }^{131}$ X. Y. Li, X.-S. Qian, S. G. Lu, J. P. Cheng, Z. Fang, and Q. M. Zhang, Appl. Phys. Lett. 99, 052907 (2011).

${ }^{132}$ G. Sebald, L. Seveyrat, J.-F. Capsal, and P.-J. Cottinet, Appl. Phys. Lett. 101, 022907 (2012).

${ }^{133}$ D. Guo, J. Gao, Y.-J. Yu, S. Santhanam, G. K. Fedder, A. J. H. McGaughey, and S. C. Yao, Appl. Phys. Lett. 105, 031906 (2014).

${ }^{134}$ G. Z. Zhang, Q. Li, H. M. Gu, S. L. Jiang, K. Han, M. R. Gadinski, M. A. Haque, Q. M. Zhang, and Q. Wang, Adv. Mater. 27, 1450 (2015).

${ }^{135}$ Z. Y. Jiang, X. C. Zheng, and G. P. Zheng, RSC Adv. 5, 61946 (2015).

${ }^{136}$ Q. Li, G. Z. Zhang, X. S. Zhang, S. L. Jiang, Y. K. Ye, and Q. Wang, Adv. Mater. 27, 2236 (2015).

${ }^{137}$ L. Yang, X. S. Qian, C. M. Koo, Y. Hou, T. Zhang, Y. Zhou, M. R. Lin, J.-H. Qiu, and Q. M. Zhang, Nano Energy 22, 461 (2016).

${ }^{138}$ S. Kar-Narayan, S. Crossley, X. Moya, V. Kovacova, J. Abergel, A. Bontempi, N. Baier, E. Defay, and N. D. Mathur, Appl. Phys. Lett. 102, 032903 (2013).

${ }^{139}$ B. Rožič, B. Malič, H. Uršič, J. Holc, M. Kosec, B. Neese, and Q. M. Zhang, Ferroelectrics 405, 26 (2010).

${ }^{140}$ V. K. Pecharsky and K. A. Gschneidner, Jr., J. Appl. Phys. 86, 565 (1999).

${ }^{141}$ V. K. Pecharsky and K. A. Gschneidner, Jr., J. Appl. Phys. 86, 6315 (1999).

${ }^{142}$ H. Yao, K. Ema, and C. W. Garland, Rev. Sci. Instrum. 69, 172 (1998).

${ }^{143}$ D. V. Christensen, R. Bjørk, K. K. Nielsen, C. R. H. Bahl, A. Smith, and S. Clausen, J. Appl. Phys. 108, 063913 (2010).

${ }^{144}$ E. Vives, S. Burrows, R. S. Edwards, S. Dixon, L. Manosa, A. Planes, and R. Romero, Appl. Phys. Lett. 98, 011902 (2011).

${ }^{145}$ C. Bechtold, C. Chluba, R. Lima de Miranda, and E. Quandt, Appl. Phys. Lett. 101, 091903 (2012).
${ }^{146}$ J. Cui, Y. M. Wu, J. Muehlbauer, Y. H. Hwang, R. Radermacher, S. Fackler, M. Wuttig, and I. Takeuchi, Appl. Phys. Lett. 101, 073904 (2012).

${ }^{147}$ H. Ossmera, F. Lambrechta, M. Gültiga, C. Chlubab, E. Quandtb, and M. Kohla, Acta Mater. 81, 9 (2014).

${ }^{148}$ J. Tušek, K. Engelbrecht, L. P. Mikkelsen, and N. Pryds, J. Appl. Phys. 117, 124901 (2015).

${ }^{149}$ Y. Liu, B. Dkhil, and E. Defay, "Spatially resolved imaging of electrocaloric effect and the resultant heat flux in multilayer capacitors," ACS Energy Lett. (submitted).

${ }^{150}$ D. Delpueyo, X. Balandraud, and M. Grédiac, Mater. Sci. Eng., A 528, 8249 (2011).

${ }^{151}$ F. Le Goupil, A. K. Axelsson, L. J. Dunne, M. Valant, G. Manos, T. Lukasiewicz, J. Dec, A. Berenov, and N. M. Alford, Adv. Energy Mater. 4, 1301688 (2014)

${ }^{152}$ Y. Bai, D. Wei, and L.-J. Qiao, Appl. Phys. Lett. 107, 192904 (2015).

${ }^{153}$ S. Kar-Narayan and N. D. Mathur, Appl. Phys. Lett. 95, 242903 (2009).

${ }^{154}$ S. Crossley, J. R. McGinnigle, S. Kar-Narayan, and N. D. Mathur, Appl. Phys. Lett. 104, 082909 (2014).

${ }^{155}$ Y. D. Wang, S. J. Smullin, M. J. Sheridan, Q. Wang, C. Eldershaw, and D. E. Schwartz, Appl. Phys. Lett. 107, 134103 (2015).

${ }^{156}$ B. Kwon, I.-J. Roh, S.-H. Baek, S. K. Kim, J.-S. Kim, and C.-Y. Kang, Appl. Phys. Lett. 104, 213902 (2014).

${ }^{157}$ M. Quintero, L. Ghivelder, F. Gomez-Marlasca, and F. Parisi, Appl. Phys. Lett. 99, 232908 (2011).

${ }^{158}$ U. Tomc, J. Tušek, A. Kitanovski, and A. Poredoš, Appl. Therm. Eng. 58, 1 (2013).

${ }^{159}$ M. M. Vopson, Solid State Commun. 152, 2067 (2012).

${ }^{160}$ A. Majumdar, Annu. Rev. Mater. Sci. 29, 505 (1999).

${ }^{161}$ S. Gomès, A. Assy, and P.-O. Chapuis, Phys. Status Solidi A 212, 477 (2015).

${ }^{162}$ F. D. Morrison, P. Zubko, D. J. Jung, and J. F. Scott, Appl. Phys. Lett. 86, 152903 (2005)

${ }^{163}$ J. F. Scott, J. Phys.: Condens. Matter 27, 492001 (2015).

${ }^{164}$ M.-W. Moon, S. H. Lee, J.-Y. Sun, K. H. Oh, A. Vaziri, and J. W. Hutchinson, Proc. Natl. Acad. Sci. U. S. A. 104, 1130 (2007).

${ }^{165}$ W. Ramberg and W. R. Osgood, "Description of stress-strain curves by three parameters," Technical Note 902, National Advisory Board for Aeronautics, Washington, DC, 1943.

${ }^{166}$ D. Matsunami and A. Fujita, Appl. Phys. Lett. 106, 042901 (2015).

${ }^{167}$ E. Defay, S. Crossley, S. Kar-Narayan, X. Moya, and N. D. Mathur, Adv. Mater. 25, 3337 (2013)

${ }^{168}$ X.-Z. Chen, X. Y. Li, X. S. Qian, M. R. Lin, S. Wu, Q. D. Shen, and Q. M. Zhang, Polymer 54, 5299 (2013).

${ }^{169}$ G. Z. Zhang, X. S. Zhang, T. N. Yang, Q. Li, L.-Q. Chen, S. L. Jiang, and Q. Wang, ACS Nano 9, 7164 (2015).

${ }^{170}$ Q. Li, L. Chen, M. R. Gadinski, S. Zhang, G. Zhang, H. Li, A. Haque, L. Q. Chen, T. Jackson, and Q. Wang, Nature (London) 523, 576 (2015).

${ }^{171}$ H. H. Wu, J. Zhu, and T. Y. Zhang, Phys. Chem. Chem. Phys. 17, 23897 (2015).

${ }^{172}$ M. Liu and J. Wang, Sci. Rep. 5, 7728 (2015).

${ }^{173}$ R. Herchig, C.-M. Chang, B. K. Mani, and I. Ponomareva, Sci. Rep. 5, 17294 (2015)

${ }^{174}$ G. Zhang, X. Zhang, H. Huang, J. Wang, Q. Li, L. Q. Chen, and Q. Wang, Adv. Mater. 28, 4811-4816 (2016).

${ }^{175}$ Y. Liu, L. C. Phillips, R. Mattana, M. Bibes, A. Barthélémy, and B. Dkhil, Nat. Commun. 7, 11614 (2016). 